\title{
A conserved strategy for inducing appendage regeneration in moon jellyfish, Drosophila, and mice
}

\author{
Michael J. Abrams ${ }^{1}+\dagger$, Fayth $\operatorname{Tan}^{1} \uparrow$, Yutian $\mathrm{Li}^{1} \uparrow$, \\ Ty Basinger ${ }^{1}$, Martin L. Heithe ${ }^{1}$, Anish A. Sarma ${ }^{1}$, Iris T. Lee ${ }^{1}$, Zevin J. Condiotte ${ }^{1}$, \\ Misha Raffiee ${ }^{1}+$, John O. Dabiri ${ }^{2}$, David A. Gold ${ }^{1}+$, Lea Goentoro ${ }^{1} *$ \\ ${ }^{1}$ Division of Biology and Biological Engineering, California Institute of Technology, Pasadena, CA \\ 91125 \\ ${ }^{2}$ Graduate Aerospace Laboratories and Mechanical Engineering, California Institute of Technology, \\ Pasadena, CA 91125 \\ $\uparrow$ These authors contributed equally to the work. \\ + Current addresses: Department of Molecular and Cell Biology, University of California at Berkeley \\ (M.J.A.), Department of Bioengineering, Stanford University (M.R.), Department of Biology and Allied \\ Health Sciences, Bloomsburg University (T.B.), Department of Earth and Planetary Sciences, University \\ of California at Davis (D.A.G) \\ * Correspondence: goentoro@ caltech.edu
}

Alternative title: Amino acid and sugar supplement induces appendage regeneration in cnidarian, insect, and mammal

Key words: Regeneration, appendage, limb, amino acid, leucine, sugar, insulin, jellyfish, Drosophila, mouse

\begin{abstract}
Can limb regeneration be induced? Few have pursued this question, and an evolutionarily conserved strategy has yet to emerge. This study reports a strategy for inducing regenerative response in appendages, which works across three species that span the animal phylogeny. In Cnidaria, the frequency of appendage regeneration in the moon jellyfish Aurelia was increased by feeding with the amino acid L-leucine and the growth hormone insulin. In insects, the same strategy induced tibia regeneration in adult Drosophila. Finally, in mammals, L-leucine and sucrose administration induced digit regeneration in adult mice, including dramatically from mid-phalangeal amputation. The conserved effect of L-leucine and insulin/sugar suggests a key role for energetic parameters in regeneration induction. The simplicity by which nutrient supplementation can induce appendage regeneration provides a testable hypothesis across animals.
\end{abstract}

\section{Introduction}

In contrast to humans' poor ability to regenerate, the animal world is filled with seemingly Homeric tales: a creature that regrows when halved or a whole animal growing from a small body piece. Two views have historically prevailed as to why some animals regenerate better than others (Goss, 1992). Some biologists, including Charles Darwin and August Weismann, hold that regeneration is an adaptive property of a specific organ (Polezhaev, 1972). For instance, some lobsters may evolve the ability to regenerate claws because they often lose them in fights and food foraging. Other biologists, including Thomas Morgan, hold that regeneration is not an evolved trait of a particular organ, but inherent in all organisms (Morgan, 1901). Regeneration evolving for a particular organ versus regeneration being organismally inherent is an important 
distinction, as the latter suggests that the lack of regeneration is not due to the trait never having evolved, but rather due to inactivation - and may therefore be induced. In support of Morgan's view, studies in past decades have converged on one striking insight: many animal phyla have at least one or more species that regenerate body parts (Sanchez-Alvarado, 2000; Bely and Nyberg, 2010). Further, even in poorly regenerative lineages, many embryonic and larval stages can regenerate. In regenerating animals, conserved molecular events (e.g., Cary et al., 2019, Kawakami et al., 2006) and regeneration-responsive enhancers (Wang et al., 2020) were identified. Although the hypothesis of convergent evolution cannot be fully excluded (e.g., Lai and Aboobaker, 2018), these findings begin to build the case that the ability to regenerate may be ancestral (Sanchez-Alvarado, 2000; Bely and Nyberg, 2010). Regeneration being possibly ancestral begs the question: is there a conserved mechanism to activate regenerative state?

This study explored how, and whether, limbs can be made to regenerate in animals that do not normally show limb regeneration. In adult frogs, studies from the early $20^{\text {th }}$ century and few recent ones have induced various degrees of outgrowth in the limb using strategies including repeated trauma, electrical stimulation, local progesterone delivery, progenitor cell implantation, and Wnt activation (Carlson, 2007; Lin et al., 2013; Kawakami et al., 2006). Wnt activation restored limb development in chick embryos (Kawakami et al., 2006), but there are no reports of postnatal regeneration induction. In salamanders, a wound site that normally just heals can be induced to grow a limb by supplying nerve connection and skin graft from the contralateral limb (Endo et al., 2004), or by delivery of Fgf2, 8, and Bmp2 to the wound site followed by retinoic acid (Viera et al., 2019). In neonatal and adult mouse digits, a model for exploring limb regeneration in mammals, bone outgrowth or joint-like structure can be induced via local implantation of Bmp2 (bone) or Bmp9 (joint; Yu et al., 2019). Thus far, different strategies gain tractions in different species, and a common denominator appears elusive.

However, across animal phylogeny, some physiological features show interesting correlation with regenerative ability (Hariharan et al., 2015; Vivien et al., 2016; Sousounis et al., 2014). First, regeneration especially in vertebrates tends to decrease with age, with juveniles and larvae more likely to regenerate than adults. For instance, the mammalian heart rapidly loses the ability to regenerate after birth and anurans cease to regenerate limbs upon metamorphosis. Second, animals that continue to grow throughout life tend to also regenerate. For instance, most annelids continue adding body segments and regenerate well, a striking exception of which is leeches that make exactly 32 segments and one of the few annelids that do not regenerate body segments (Rouse, 1998). Consistent with the notion of regeneration as ancestral, indeterminate growth is thought of as the ancestral state (Hariharan et al., 2015). Finally, a broad correlate of regenerative ability across animal phylogeny is thermal regulation. Poikilotherms, which include most invertebrates, fish, reptiles and amphibians, tend to have greater regenerative abilities than homeotherms - birds and mammals are animal lineages with poorest regeneration. These physiological correlates, taken together, are united by the notion of energy expenditure. The transition from juvenile to adult is a period of intense energy usage, continued growth is generally underlined by sustained anabolic processes, and regulating body temperature is energetically expensive compared to allowing for fluctuation. Regeneration itself entails activation of anabolic processes to rebuild lost tissues (Hirose et al., 2014; Naviaux et al., 2009; Malandraki-Miller et al., 2018). These physiological correlates thus raise the notion of a key role of energetics in the evolution of regeneration in animals. Specifically, we wondered whether energy inputs can promote regenerative state. In this study, we demonstrate that nutrient 
supplementation can induce regenerative response in appendage and limb across three vastly divergent species.

\section{Results}

91 Leucine and insulin promote appendage regeneration in the moon jelly Aurelia

We reasoned that if there was an ancestral mechanism to promote regeneration, it would likely remain intact in early-branching lineages with prevalent regeneration across the species. In Cnidaria, the ability to regenerate is established in polyps, e.g., hydras and sea anemones. Some cnidarians, notably jellyfish, not only exist as sessile polyps, but also as free-swimming ephyrae and medusae (Figure 1a). In contrast to the polyps' ability to regenerate, regeneration in ephyrae and medusae appears more restricted in some species (Abrams et al., 2015; Sinigaglia et al 2020; Schmid and Alder, 1984). We focused on the moon jellyfish Aurelia coerulea (formerly A. aurita sp. 1 strain), specifically on the ephyra, whose eight arms facilitate morphological tracking (Figure 1b). About 3 millimeter in diameter, Aurelia ephyrae regenerate tips of arms and the distal sensory organ rhopalium, but upon more dramatic amputations such as removing a

102

103 whole arm or halving the body, rapidly reorganize existing body parts and regain radial symmetry (Figure 1c). Observed across four scyphozoan species, symmetrization occurs rapidly within 1-3 days and robustly across conditions (Abrams et al., 2015). Ephyrae that symmetrized matured into medusae, whereas ephyrae that failed to symmetrize and simply healed the wound

106

107 grew abnormally.
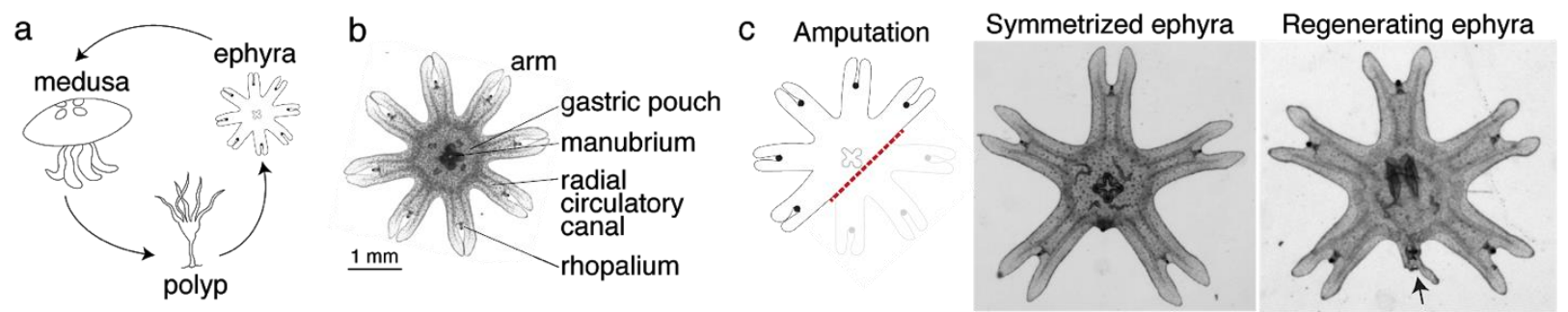

Figure 1. Aurelia as a system to identify factors that promote appendage regeneration.

110

111

112

113

114

115

116

117

118

(a) The moon jellyfish Aurelia aurita have a dimorphic life cycle, existing as sessile polyps or freeswimming medusae and ephyrae. Ephyra is the juvenile stage of medusa, a robust stage that can withstand months of starvation. In lab conditions, ephyrae mature into medusae, growing bell tissue and reproductive organs, in 1-2 months.

(b) Ephyrae have eight arms, which are swimming appendages that contract synchronously to generate axisymmetric fluid flow, which facilitates propulsion and filter feeding. The eight arms are symmetrically positioned around the stomach and the feeding organ manubrium. Extending into each arm is radial muscle (shown in Figure 2) and a circulatory canal that transports nutrients. At the end of each arm is the light- and gravity-sensing organ rhopalium.

(c) In response to injury, the majority of ephyrae rapidly reorganize existing body parts and regain radial symmetry. However, performing the experiment in the natural habitat, a few ephyrae (2 of 18) regenerated a small arm (arrow).

Intriguingly, in the course of our previous study (Abrams et al., 2015), we observed in a few symmetrizing ephyrae, a small bud at the amputation site. To follow this hunch, we repeated the experiment in the original habitat of our lab's polyp population, off the coast of Long Beach, CA (Methods). Two weeks after amputation, most ephyrae indeed symmetrized, but in 2 of 18 animals a small arm grew (Figure 1c). This observation suggests that, despite symmetrization 
being the more robust response to injury, an inherent ability to regenerate arm is present and can 128 be naturally manifest. The inherent arm regeneration presents an opportunity: Can arm regeneration be reproduced in the lab, as a way to identify factors that promote regenerative state?

To answer this question, we screened various molecular and physical factors (Figure 2a, Figure 2-figure supplement 1). Molecularly, we tested modulators of developmental signaling pathways as well as physiological pathways such as metabolism, stress response, immune and inflammatory response. Physically, we explored environmental parameters, such as temperature, oxygen level, and water current. Amputation was performed across the central body removing 3 arms (Figure 2a). Parameter changes were implemented or molecular modulators (e.g., peptides, small molecules) were introduced into the water immediately after amputation. Regenerative response was assessed for 1-2 weeks until the onset of bell growth, which hindered the scoring of arm regeneration (Figure 2-figure supplement 2).
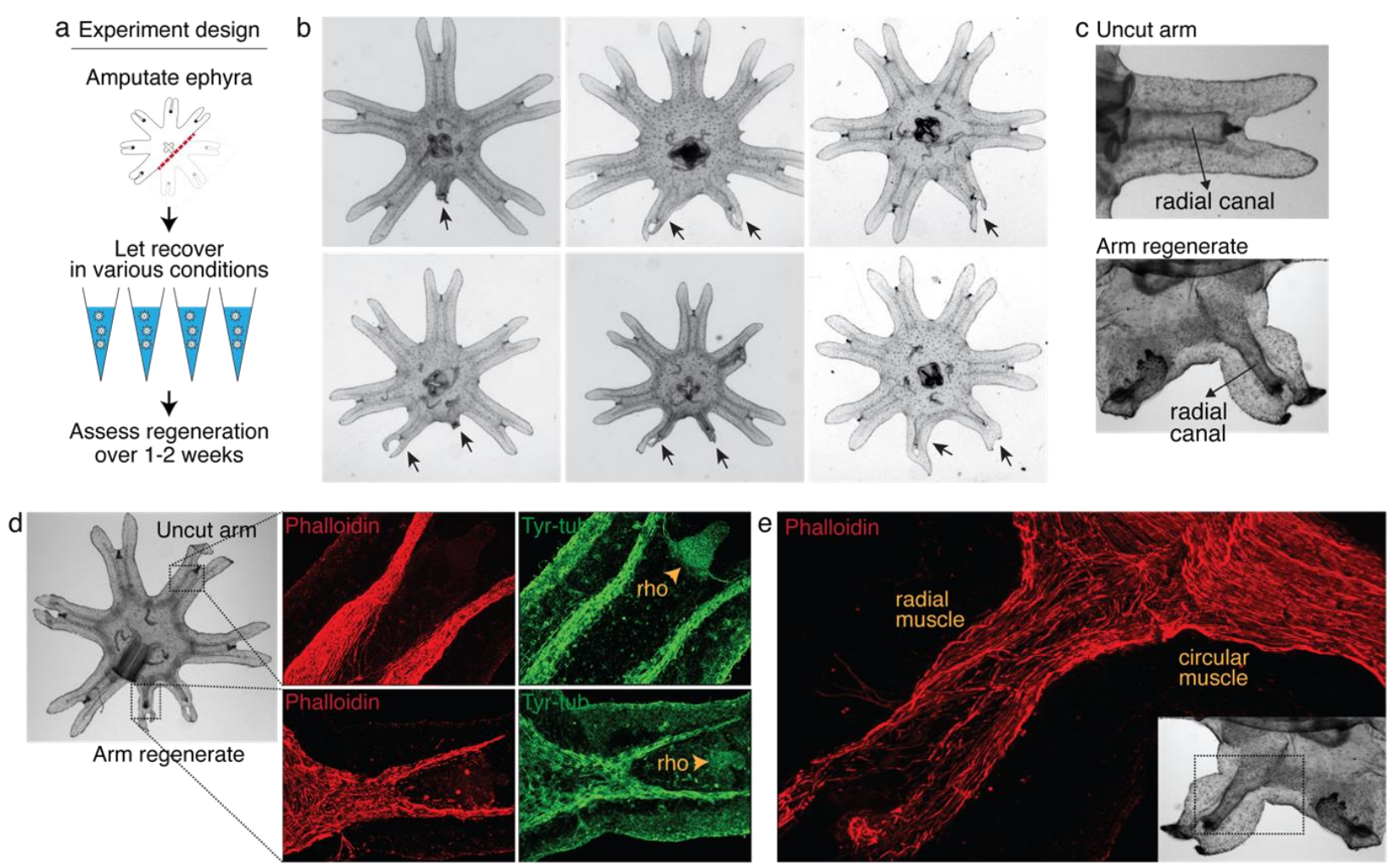

Figure 2. Arm regeneration in Aurelia ephyra can be induced using exogenous factors.

142 (a) Ephyrae were amputated (red line) across the body to remove 3 arms, and then let recover in various 143 conditions. Figure 2-figure supplement 1 tabulates the factors tested in the screen. Regeneration was 144 assessed over 1-2 weeks until bell tissues began developing between the arms and obscured scoring. The 145 ephyrae shown are from high-nutrient condition (see Figure 3).

146 (b) Arm regeneration (arrows; from high food condition, see Figure 3a).

147 (c) Radial circulatory canal in an uncut arm and is reformed in an arm regenerate.

148 (d) Muscle (red), as indicated by phalloidin staining, and neuronal networks (green), as indicated by 149 antibody against tyrosinated tubulin. The orange arrows indicate distal enrichment of tyrosinated-tubulin 150 staining, which marks the sensory organ rhopalium (rho). Twenty ephyrae were examined and 151 representative images are shown. 
152

153

154

155

156

157

158

159

160

161

162

163

164

165

166

167

168

169

170

171

172

173

174

175

176

177

178

179

180

181

182

183

184

185

186

187

188

189

190

191

192

193

194

195

196

(e) Higher magnification of the phalloidin staining shows the striated morphology of the regrown muscle in the arm regenerate (called radial muscle), which extends seamlessly from circular muscle in the body. 3 supplements: Figure 2-figure supplement 1-3. Source file: Aurelia screen.xls

After 3 years of screening, only three factors emerged that strongly promoted arm regeneration (Figure 2b). The ephyrae persistently symmetrized in the majority of conditions tested. In the few conditions where regeneration occurred, arm regenerates show multiple tissues regrown in the right locations: circulatory canals, muscle, neurons, and rhopalium (Figure 2c-e). The arm regenerates contracted synchronously with the original arms (Video 1), demonstrating a functional neuromuscular network. Thus, arm regeneration in Aurelia that was observed in the natural habitat can be recapitulated in the lab by administering specific exogenous factors.

The extent of arm regeneration varied, from small to almost fully sized arms (Figure 2b). The variation manifested even within individuals: a single ephyra could grow differently sized arms. Of the three arms removed, if regeneration occurred, generally one arm regenerated (67\%), occasionally 2 arms (32\%), and rarely 3 arms (1\%, of the 4270 total ephyrae quantified in this study). Finally, the frequency of regeneration varied across clutches, i.e., strobilation cohorts. Some variability may be due to technical factors, e.g., varying feed culture conditions; however, variability persisted even with the same feed batch. We verified that the variability was not entirely due to genetic differences, as it manifested across clonal populations (Figure 2-figure supplement 3). Thus, there appears to be stochasticity in the occurrence of arm regeneration in Aurelia and the extent to which regeneration proceeds.

What are the factors that promote arm regeneration? Notably, modulation of developmental pathways often implicated in regeneration literature (e.g., Wnt, Bmp, Tgfß) did not produce effect in the screen (Figure 2-figure supplement 1) -although we do not rule out their involvement in other capacity, e.g., in downstream patterning. We first identified a necessary condition: water current. The requirement for current for promoting regeneration is interesting because ephyrae can recover from injury by symmetrizing in stagnant water (Figure 1c). Thus, a specific physiological state is required for enabling regenerative response. Behaviorally, the presence of current promotes more swimming, while in stagnant water ephyrae tend to rest at the bottom and pulse stationarily (Figure 3-figure supplement 1 and Video 2 show the aquarium setup used to implement current). In this permissive condition, the first factor that promotes regeneration is the nutrient level: increasing food amount increases the frequency of arm regeneration. To measure the regeneration frequency, we scored any regenerates with lengths greater than $15 \%$ of that of an uncut arm (Figure 3a). This threshold was chosen to predominantly exclude non-specific growths or buds that show no morphological structures (Figure $3 \mathrm{~b}$ ) while including small arm regenerates that show clear morphological features, i.e., lappets, radial canal, and radial muscle sometimes showing growing ends (Figure 3b). Given the clutch-to-clutch variability, control and treatment were always performed side by side using ephyrae from the same clutch. The effect size of a treatment was assessed by computing the change in regeneration frequency relative to the internal control. Statistical significance of a treatment was assessed by evaluating the reproducibility of its effect size across independent experiments (Methods). With these measurement and statistical methodologies, we found that although the baseline regeneration frequency varied across clutches, higher food amounts reproducibly increased regeneration frequency (Figure 3c). The magnitude of the increase varied (Figure 3g, 95\% CI [4.7, 12.1-fold]), but the increase was reproducible (95\% CI excludes 1) and statistically significant $\left(\mathrm{p}\right.$-value $\left.<10^{-4}\right)$. 
The second factor that promotes regeneration is insulin (Figure 3d). We verified that the 198 insulin receptor is conserved in Aurelia (Figure 3-figure supplement 2). Administering insulin 199 led to a reproducible (Figure 3g, 95\% CI [1.1, 5.0-fold]) and statistically significant (p200 value $<0.05)$

a Quantitation protocol

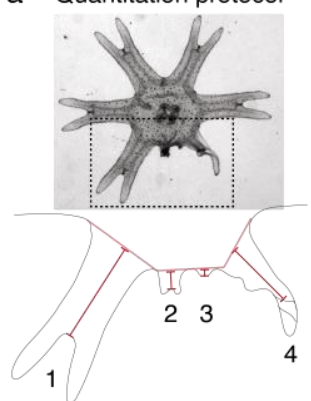

1: Uncut arm

2: $0.3 x$ length of uncut arm 3: $0.06 x$ length of uncut arm 4: $0.5 x$ length of uncut arm Scoring threshold: $>0.15 x$ b
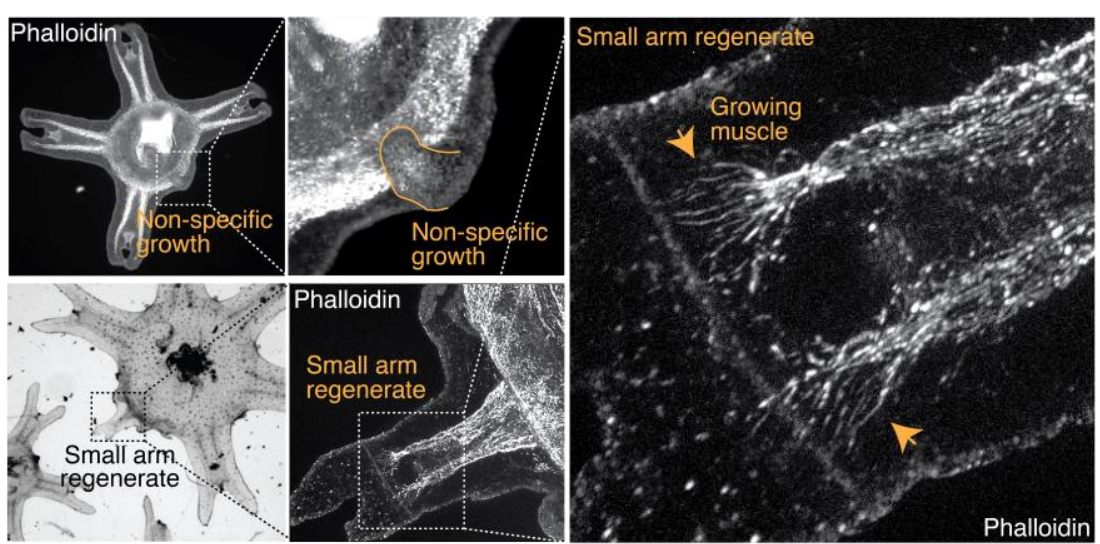

c

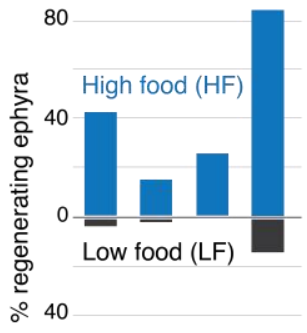

80

$$
\begin{array}{|rcccc}
\hline \text { Exp } & 1 & 2 & 3 & 4 \\
\hline \% & 2.6 & 1.2 & 0 & 13.8 \\
43.2 & 15.1 & 25.6 & 85.1 \\
& 43 & \\
\text { N } & 78 & 82 & 49 & 87 \\
& 74 & 86 & 82 & 87
\end{array}
$$

d
80

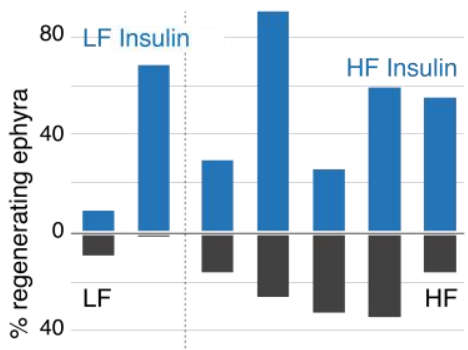

80

$\begin{array}{llllllll}\operatorname{Exp} & 1 & 2 & 3 & 4 & 5 & 6 & 7\end{array}$

$\begin{array}{llllllll}\% & 8.2 & 1.1 & 15.1 & 25.6 & 31.9 & 34.0 & 15.1\end{array}$ $\begin{array}{lllllll}8.2 & 68.6 & 29.3 & 90.9 & 25.6 & 59.3 & 55.3\end{array}$

$\begin{array}{llllllll}\mathbf{N} & 85 & 88 & 86 & 82 & 69 & 103 & 26\end{array}$ $\begin{array}{lllllll}85 & 80 & 82 & 77 & 82 & 91 & 38\end{array}$ e

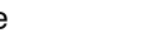

Hypoxia

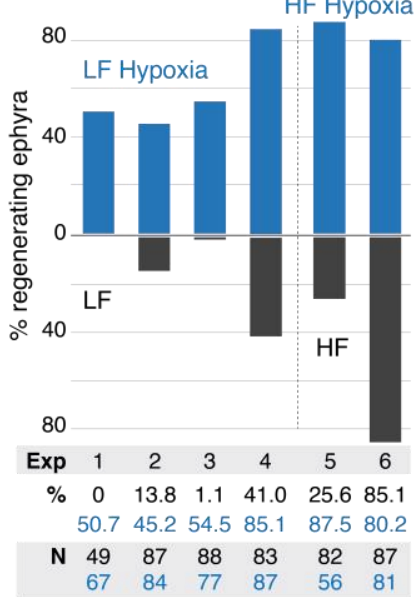

f Leucine

80

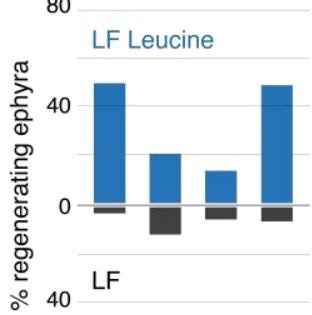

Legend

HF High food Exp Experiment number

LF Low food $\quad \%$ Percentage of regenerating ephyrae in control (black) and treated (blue) groups $\mathbf{N}$ Number of ephyrae examined in the control (black) and treated (blue) groups

g

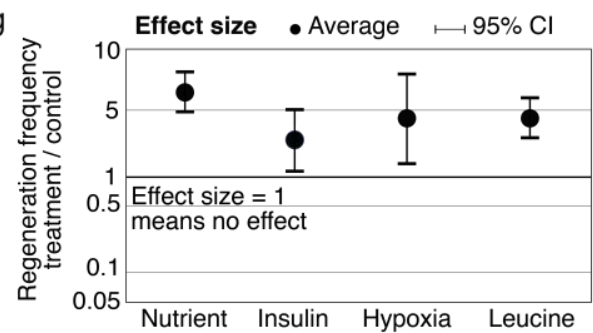

\begin{tabular}{|lcclll|}
\hline Treatment & $\begin{array}{c}\text { Average } \\
\text { effect size }\end{array}$ & $\mathbf{9 5 \%} \mathbf{C l}$ & p-value & \\
Nutrient & 7.5 & $4.7,12.1$ & $<0.0001$ & $* * *$ \\
Insulin & 2.4 & $1.1,5.0$ & 0.023 & $*$ \\
Hypoxia & 4.1 & $1.4,12.0$ & 0.0099 & $* *$ \\
Leucine & 4.1 & $2.5,6.6$ & $<0.0001$ & $* * *$ \\
\hline
\end{tabular}

Figure 3. Nutrient level, insulin, hypoxia, and leucine increased regeneration frequency in Aurelia.

(a) An ephyra is regenerating if it has at least one growth from the cut site with a length greater than 0.15 of the uncut arm length. The uncut arm length was determined in each ephyra by measuring 3 uncut arms and taking the average. Lappets, the distal paired flaps, were excluded in the length measurement because their shapes tend to vary across ephyrae. The measurements were performed in ImageJ. 
208

209

210

211

212

213

214

215

216

217

218

219

220

221

222

223

224

225

226

227

228

229

230

231

232

233

234

235

236

237

238

239

240

241

242

243

244

245

246

247

248

249

250

251

252

253

254

(b) The threshold 0.15 was chosen to balance excluding non-specific growths that show no morphological structures (e.g., as shown, lack of phalloidin-stained structures) and retaining rudimentary arms that show morphological structures, including radial muscle sometime with growing ends (shown, phalloidin stained).

(c-f) In each experiment, treated (blue) and control (grey) ephyrae came from the same strobilation.

(c) Regeneration frequency in lower amount of food (LF) and higher amount of food (HF). The designation "high" and "low" is for simplicity, and does not presume the nutrient level in the wild. If we were to speculate, the LF amount is likely closer to typical nutrient level in the wild, based on two lines of evidence. First, regeneration frequency in LF is comparable to that observed in the natural habitat experiment. Second, in many of the wild populations studied, ephyrae mature to medusae over 1-3 months (Lucas, 2001), comparable to the growth rate in LF (by contrast, ephyrae in HF mature to medusae over 3-4 weeks).

(d) Regeneration frequency in $500 \mathrm{nM}$ insulin.

(e) Regeneration frequency in ASW with reduced oxygen.

(f) Ephyrae recovering in low food, with or without $100 \mathrm{mM}$ L-leucine.

(g) The effect size of a treatment was computed from the ratio between regeneration frequency in treated and control group within an experiment, i.e., the metric Risk Ratio (RR; RR $=1$ means the treatment has no effect [Borenstein et al., 2009]). The statistical significance and reproducibility of a treatment was assessed by analyzing the effect size across experiments using the meta-analysis package, metafor (Viechtbauer 2010), in R with statistical coefficients based on normal distribution. See Methods for details. A treatment was deemed reproducible if the 95\% confidence intervals (95\% CI) of RR exclude 1. The p-value evaluates the null hypothesis that the estimate RR is 1 . Reproducibility and statistical significance of each treatment were verified using another common size effect metric, Odds Ratio (Figure 3 -figure supplement 3).

6 supplements: Figure 3 -figure supplement 1-7. Source files: Aurelia data.xls. Source codes: R-codes.rtf

increase in regeneration frequency. The insulin effect was unlikely to be due to non-specific addition of proteins, since bovine serum albumin at the same molarity showed no statistically significant effect (Figure 3-figure supplement 4). Finally, the third promoter of regeneration is hypoxia (Figure 3e). We verified that the ancient oxygen sensor $\mathrm{HIF} \alpha$ is present in Aurelia (Figure 3-figure supplement 2). Hypoxia led to a reproducible (Figure 3g, 95\% CI [1.4, 12.0fold]) and statistically significant (p-value $<0.01)$ increase in regeneration frequency. To reduce oxygen, nitrogen was flown into the seawater, achieving $\sim 50 \%$ reduction in dissolved oxygen level (Methods). We verified that the effect was due to reduced oxygen rather than increased nitrogen, since reducing oxygen using argon flow similarly increased regeneration frequency (Figure 3-figure supplement 4). The factors can act synergistically (e.g., insulin and high nutrient level), but the effect appears to eventually saturate (e.g., hypoxia and high nutrient level).

In addition to quantifying the number of ephyrae that regenerate, we further quantified the regeneration phenotypes in each ephyra, i.e., the number of arms regenerating, the length of arm regenerates, and the formation of rhopalia (Figure 3-figure supplements 5 and 6). Nutrient level strikingly improved all phenotypic metrics: not only more ephyrae regenerated in higher nutrients, more ephyrae regenerated multiple arms, longer arms, and arms with rhopalia. Insulin and hypoxia, interestingly, show differential phenotypes. Most strikingly, while insulin induced more ephyrae to regenerate multiple arms, hypoxia induced largely single-arm regenerates, e.g., hypoxia experiments 3 and 5 in Figure 3-figure supplement 5c. Thus, while all factors increased the probability to regenerate, they had differential effects on the regeneration 
phenotypes, suggesting a decoupling to a certain extent between the regulation of the decision to regenerate and the regulation of the subsequent morphogenesis.

Of the three factors identified in the screen, nutrient input is the broadest, and prompted us to search if a more specific nutritional component could capture the effects of full nutrients in promoting regeneration. Jellyfish are carnivorous and eat protein-rich diets of zooplanktons and other smaller jellyfish (Graham and Kroutil, 2001). Notably, all three factors induced growth: treated ephyrae are larger than control ephyrae (Figure 3-figure supplement 7). The growth effect is interesting because of essential amino acids that must be obtained from food, branched amino acids supplementation correlates positively with protein synthesis and growth, and in particular, L-leucine appears to recapitulate most of the anabolic effects of high amino acid diet (Lynch and Adams, 2001; Stipanuk, 2007). Motivated by the correlation between growth and increased regeneration frequency, we wondered if leucine administration could promote regeneration. Animals typically have a poor ability to metabolize leucine, such that the extracellular concentrations of leucine fluctuate with dietary consumption (Wolfson et al., 2016). As a consequence, dietary leucine directly influences cellular metabolism. Feeding amputated ephyrae with leucine indeed led to increased growth (Figure 3-figure supplement 6). Assessing arm regeneration in the leucine-supplemented ephyrae, we observed a significant increase in the regeneration frequency (Figure 3f-g, 95\% CI [2.5, 6.6-fold], p-value $<10^{-4}$ ). Furthermore, leucine treatment phenocopies the effect of high nutrients, improving all measured phenotypic metrics: increasing multi-arm regeneration, the length of arm regenerate, and the frequency of rhopalia formation (Figure 3-figure supplement 5-6).

These experiments demonstrate that abundant nutrients, the growth factor insulin, reduced oxygen level, and the amino acid L-leucine promote appendage regeneration in Aurelia ephyra. The identified factors are fundamental physiological factors across animals. Might the same factors promote appendage regeneration in other animal species?

\section{Leucine and insulin induce regeneration in Drosophila limb}

To pursue this question, we searched for other poorly regenerating systems, which fortunately include most laboratory models. Drosophila, along with beetles and butterflies, belong to the holometabolans - a vast group of insects that undergo complete metamorphosis, and that as whole, do not regenerate limbs or other appendages as adults (Hopkins and Das, 2015). Larval stages have imaginal disks, undifferentiated precursors of adult appendages such as the legs and antennae, and portions of imaginal disks have been shown to regenerate (Worley et al., 2012). Motivated by findings in Aurelia, we asked if leucine and insulin administration can induce regenerative response in the limb of adult Drosophila. We focused on testing leucine and insulin in this study because of considerations of specificity (i.e., nutrients are broad and composition of nutritional needs vary across species), pragmatism (i.e., administering hypoxia requires more complex setups), and in the case of Drosophila specifically, Drosophila being resistant to hypoxia (Haddad et al., 1997).

We amputated Drosophila on the hindlimb, across the fourth segment of the leg, the tibia (Figure 4a). After amputation, flies were housed in vials with standard food (control) or standard food supplemented with leucine and insulin, with glutamine to promote leucine uptake (Nicklin et al., 2009) (treated) (Figure 4c). Each fly was examined multiple times, twice in the first week, and then once weekly over the course of 2-4 weeks. 
No regrown tibia was found in the 860 control flies examined (Figure 4b-c). In the treated flies, only tibia stumps were observed in the first week after amputation. But remarkably, at 7-21 $300 \mathrm{dpa}$, a few regrown tibias were observed (1.0\%, N=387; Figure 4d-e). The regrown tibias culminate in reformed joints, articulating from which appears to be the beginning of a next

a

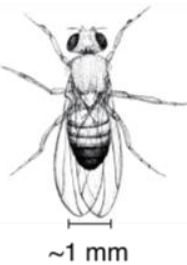

$\sim 1 \mathrm{~mm}$
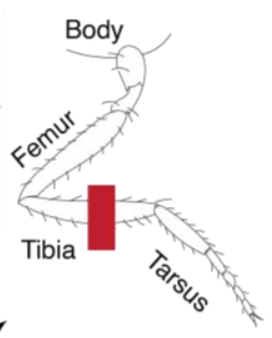

Control Treated

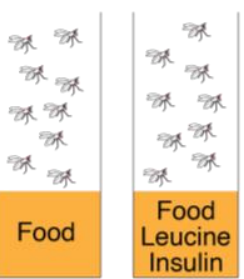

$\downarrow$

Examine each tibia stump for 3-4 weeks
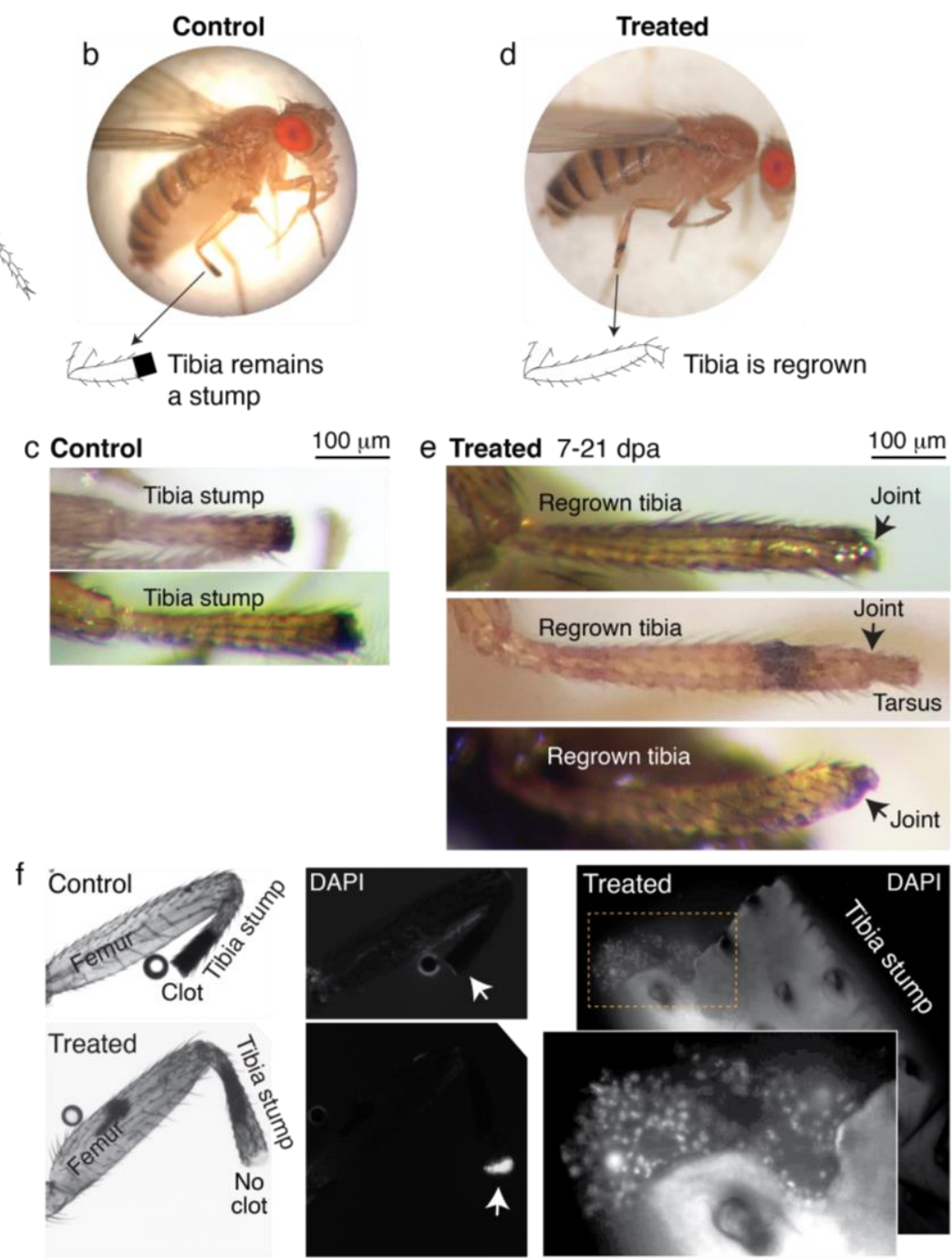

Figure 4. Leucine and insulin induced regeneration in Drosophila limb.

(a) Adult Drosophila have jointed limbs, with rigid segments connected by flexible joints. Amputation was performed across the tibial segment. After amputation, flies were housed in vials containing standard lab food (control) or standard lab food added with $1.7 \mathrm{mM}$ L-Leucine, $1.7 \mathrm{mM}$ L-Glutamine, and 33 $\mu \mathrm{g} / \mathrm{mL}$ insulin (treated). Doses were determined through observing the highest order of magnitude dose of amino acid that could be fed to flies over a prolonged period without shortening their lifespan. The flies were examined at $\sim 1,3,7,14$, and 21 days post amputation (dpa).

(b) A control fly, imaged at 7 dpa.

(c) Control tibia stumps, imaged at 1-3 dpa.

(d) A treated fly, imaged at 7 dpa.

(e) At 7-21 dpa, regrown tibias, which culminate in joints, were observed in the treated population.

(f) Tibia stumps at 3-14 dpa were dissected, fixed, and mounted in Vectashield mounting medium with DAPI. Samples from 14 dpa are shown here. Insect cuticle is not dissected to restrict DAPI penetrance only to the distal tip. Clotted tips of control tibia stumps did not stain with DAPI (10 of 10), whereas 
unclotted tips of treated tibia stumps stained with DAPI (14 of 16). Higher-resolution confocal image of an unclotted treated tibia stump at 14 dpa.

Drosophila drawing in (a) is by Ashley Smart and used with permission.

1 supplement: Figure 4-figure supplement 1.

segment. Control tibia stumps showed melanized clots at the tips within 1-3 dpa (Figure 4b), as expected from normal wound healing process (Ramet et al., 2002), while some treated tibias showed no clot $(12.1 \%, \mathrm{~N}=387)$ and the tips stained positively with DAPI instead (14 of 16 tibias examined; Figure 4f). Induction of regenerative response was observed across genetic backgrounds, in Oregon R, as discussed, and Canton S wild-type strains (Figure 4-figure supplement 1a). Reminiscent of Aurelia, not all regenerative response was patterned, few flies showed non-specific outgrowth (Figure 4-figure supplement 1b).

The bulk experiment enables us to assess a large number of flies and can capture dramatic regenerative response, i.e., a fully regrown leg segment. But it can miss partial segment regrowth and therefore underestimate the extent of regenerative response. To track regenerative response in individual flies, we housed in each vial a small number of flies that were amputated in different limbs (Figure 5a). The amputated limb position, combined with sex, enabled unique identification of each fly within a vial. Each fly was imaged immediately after amputation and 1-3 additional times over the course of 2-4 weeks. The imaging frequency balanced obtaining time-lapse information with minimizing stress from repeated anesthesia.

The single-fly tracking showed pervasive regenerative response induced by nutrient supplementation. Time-lapse pictures of select control and treated flies are shown in Figure 5bd. For each tibia stump, we measured the length over time (Figure 5e). Control tibia stumps showed near-zero percent change in length (Figure 5d; mean $-0.3 \%$, 95\% CI [-3.8, 3.2\%], $\mathrm{N}=116$ ). By contrast, $49 \%$ of treated flies showed growth beyond the $95 \%$ confidence intervals of the control distribution (Figure 5f; N=150). The rate of regrowth varied; Some flies showed large growth during the first 1-2 weeks post amputation, while others regrow more slowly over 2-3 weeks (Figure 5c). Interestingly, 4 flies (2.7\%) showed shortened tibia stumps, which may indicate roles of histolysis in regenerative response. Regenerative response was not only observed from amputations across the tibia, but also from more dramatic amputations across the femur (Figure 5d). We verified that the control and treated length change distributions are statistically different (p-value $<0.0001^{* * *}$, non-parametric Kruskal-Wallis test). No sex-based differences in response were observed (Figure 5-figure supplement 1a).

In some limbs, the new growth already looks almost indistinguishable from the rest of the leg. In some limbs, the new growth showed what may be intermediate morphologies. For instance, some new growths are pigmented differently and/or have no sensory bristles (Figure $5 \mathrm{~g}$, Figure 5-figure supplement 1d, e) or showed white tissues protruding from the end (e.g., Figure 5-figure supplement 1c), which was remodeled over time (e.g., Figure 5-figure supplement 1d). Finally, reproducing what was observed in the bulk experiments, 5 flies showed reformed tibia segments: showing a tapering end ( $\mathrm{N}=150$; Figure $5 \mathrm{~h}$ ), what appeared to be a joint (Figure 5i), and the beginning of the next segment (Figure 5j).

A scanning electron micrograph of a regrown hindlimb tibia (the top tibia in Figure 4e), taken one week later) morphologically confirms the regenerated joint as a tibial/tarsal joint. The joint-like structure shows the expected bilateral symmetry of a tibial/tarsal joint (as opposed to e.g., the radially symmetrical tarsal/tarsal 
a Tracking single flies
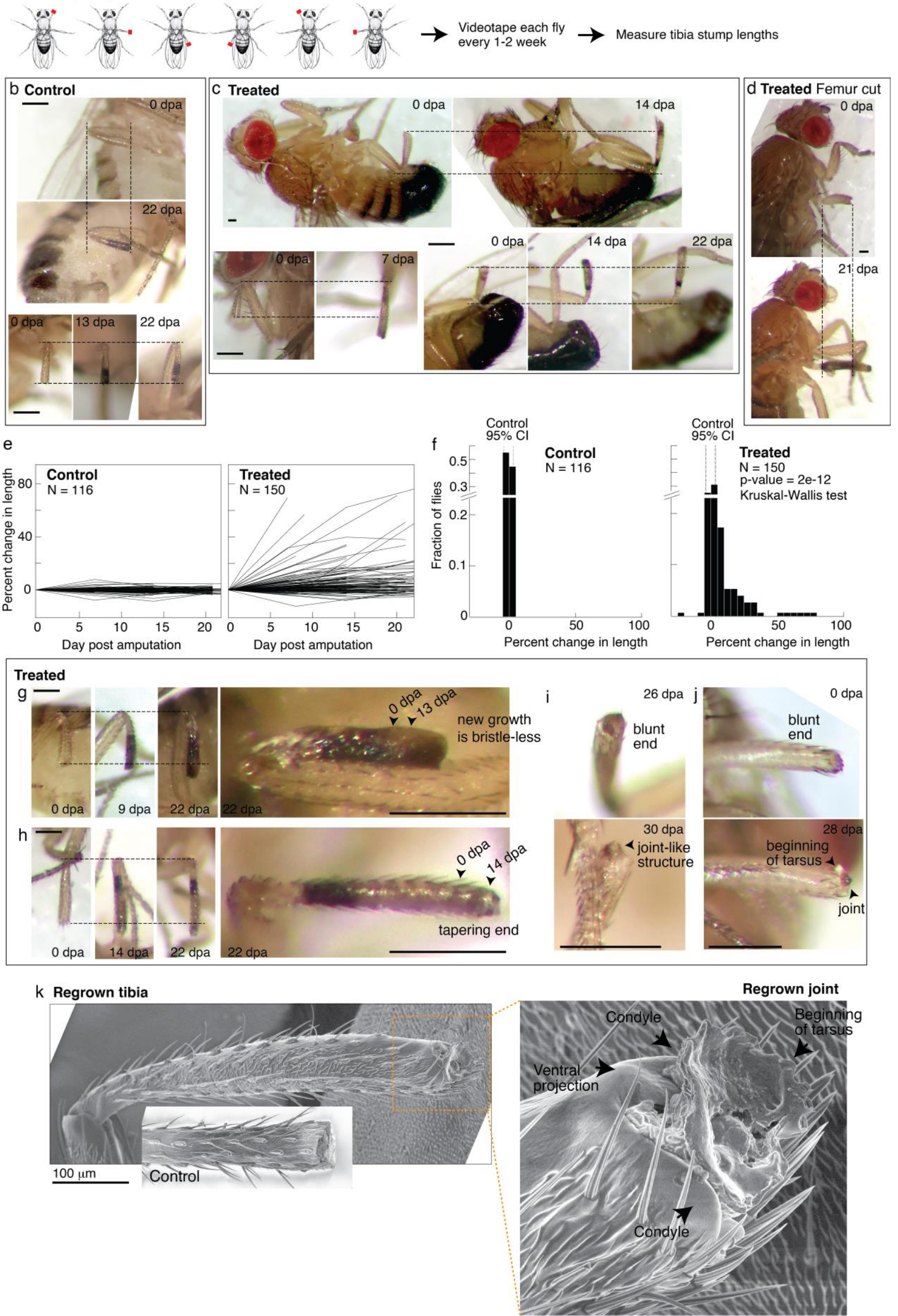
(a) To track the regenerative response in individual flies, flies were amputated in different limbs (fore, mid, hindlimb) and housed in small groups such that in any given vial, each fly was uniquely identifiable by sex and amputated limb. Each fly was imaged immediately after amputation and 1-3 additional times over the course of 2-4 weeks. (b-d) Representative time lapse from control (b) and treated flies (c-d). dpa: days post amputation. For each fly, time-lapse images are shown at the same magnification. Scale bars: $250 \mu \mathrm{m}$. (e) Change in tibia stump length over time.

(f) Distribution of change in tibia stump length in control and treated flies. Percent change in length is the difference between the length at the final time point and $0 \mathrm{dpa}$, relative to the length at $0 \mathrm{dpa}$. CI: confidence intervals. Statistical difference between control and treated distributions was evaluated using the non-parametric Kruskal-Wallis test. The p-value tests the null hypothesis that the data are drawn from the same distribution. We confirmed that the probability of observing growth in each condition was not biased by differences in the initial length of tibia stump (Figure 5-figure supplement 1b). (g-i) For each fly, time-lapse images are shown at the same magnification. Scale bars: $250 \mu \mathrm{m}$.

(g) In this treated tibia stump, the new growth showed different pigmentation and no sensory bristles.

(h) In this treated tibia stump, the segment was reformed as suggested by the tapering end.

(i) In this treated tibia stump, the segment was reformed and a joint-like structure grew.

(j) In this treated tibia stump, amputation was performed just before the joint. Four weeks later, a new joint-like structure appeared, from which tissues of the next segment (tarsus) start growing.

(k) Fly with a regrown tibia at 21 dpa was mounted onto an environmental SEM with a copper stub. Inset shows a clotted tibia stump from a control fly, with the discoloration at the end corresponding to the clot. Magnification of the regenerated joint: the arrows denote the two condyles and additional ventral projection.

Drosophila drawing in Figure a is by Ashley Smart and used with permission.

1 supplement: Figure 5-figure supplement 1. Source files: Drosophila data.xls, DrosophilaCode.m

joint) (Mirth and Akam, 2002) with rounded projections at the posterior and anterior end (arrows in Figure 5k). These projections, called condyles, function as points of articulation between opposing leg segments. Indeed, articulating from the regrown condyles appears to be further growth of the next tarsal segment. Finally, a unique feature of the tibial/tarsal joint of the hindlimb (but not of fore or midlimb) is an additional ventral projection between the side condyles (Mirth and Akam, 2002), which serves to restrain bending of the leg upward. The ventral projection is indeed present in the regenerated joint (arrow in Figure 5k). Altogether, these data demonstrate for the first time that patterned regenerative response can be induced from adult Drosophila limbs.

\section{Leucine and sucrose induce regeneration in mouse digit}

The ability of leucine and insulin to induce regenerative response in Drosophila limb and Aurelia appendage motivated testing in vertebrates. One sign that limb regeneration may be feasible in humans is that fingertips regenerate (Illingworth, 1974). The mammalian model for studying limb regeneration is the house mouse, Mus musculus, which like humans regenerates digit tips. Although proximal regions of digits do not regenerate, increasing evidence suggests that they have inherent regenerative capacity. In adult mice, implanting developmental signals in amputated digits led to specific tissue induction, i.e., bone growth with Bmp4 or joint-like structure with Bmp9 (Yu et al., 2019). In neonates, reactivation of the embryonic gene lin28 led to distal phalange regrowth ( $\mathrm{Ng}$ et al., 2013). Thus, while patterned phalange regeneration can be induced in newborns, induction in adults so far involves a fine-tuned stimulation, e.g., to 
elongate bone and then make joint, Bmp4 was first administered followed by Bmp9 in a timed manner. Motivated by the findings in Aurelia and Drosophila, we tested if leucine and insulin administration could induce a self-organized regeneration in adult mice.

We performed amputation on the hindpaw (Figure 6a), on digit 2 and 4, leaving the middle digit 3 as an internal control (Figure 6b). To perform non-regenerating amputation, a clear morphological marker is the nail, which is associated with the distal phalange (P3). Amputation that removes $<30 \%$ of $\mathrm{P} 3$ length, that cuts within the nail, readily regenerates, whereas amputation that removes $>60 \%$ of $\mathrm{P} 3$ length, corresponding to removing almost the entire visible nail, does not regenerate (Figure 6c) (Chamberlain et al., 2017; Lehoczky et al., 2011). We therefore performed amputations entirely proximal to the visible nail-giving, within the precision of our amputation, a range of cut across somewhere between the proximal P3 and the distal middle phalange (P2) (Figure 6d) - a range that is well below the regenerating tip region. Note additional morphological markers that lie within the non-regenerating region: the os hole ('o' in Figure 6c), where vasculatures and nerves enter P3, the bone marrow cavity (' $\mathrm{m}$ ' in Figure 6c), and the sesamoid bone ('s' in Figure 6c) adjacent to P2.

The digit portion removed was immediately fixed to determine the precise plane of amputation. The amputated mice were either provided with water as usual (control) or water supplemented with leucine and sucrose (treated) (Figure 6e). Both groups were monitored for 7-8 weeks. Sucrose was used because insulin is proteolytically digested in the mammalian gut. The sucrose doses used are lower or the administration duration is shorter than those shown to induce insulin resistance (Cao et al., 2007; Togo et al., 2019). We verified that control and treated mice had comparable initial weights $(35.1 \pm 0.6$ vs $34.1 \pm 1.1$ grams, p-value $=0.402$, student's t-test), and that as expected from amino acid and sugar supplementation, treated mice gained more weight over the experimental duration $(4.5 \pm 1.0$ vs $7.8 \pm 1.0$ grams, p-value $=0.028$, student's ttest).

As expected for amputation proximal to the nail, no regeneration was observed in the control mice ( $\mathrm{N}=34$ digits, 17 mice). Amputated digits healed and re-epithelialized the wound as expected (Figure 6f). Skeletal staining shows blunt-ended digit stumps (Figure 6i) and in many instances, as expected, dramatic histolysis, a phenomenon where bone recedes further from the amputation plane (Figure 6-figure supplement 1) (Chamberlain et al., 2017). By contrast, $18.8 \%$ of the treated digits ( $\mathrm{N}=48$ digits, 24 mice) showed various extents of regenerative response (Figure 6-figure supplement 1). The increase in regeneration frequency due to the treatment is statistically significant $\left(95 \% \mathrm{CI}[8,30 \%]\right.$, p-value $=0.0019,{ }^{* *}$, student's t-test).

We observed, as in Aurelia and Drosophila, an unpatterned response (Figure 6-figure supplement 1), wherein skeletal staining reveals excessive bone mass around the digit stump, similarly to what was observed in some cases with BMP stimulation (Yu et al., 2019). However, we also observed patterned responses (Figure 6-figure supplement 2). The most dramatic regenerative response was observed in 2 digits (Figure $6 \mathrm{~g}-\mathrm{h}$ ). In one digit, an almost complete regrowth of the distal phalange and the nail was observed (Figure 6g). Skeletal staining of the portion removed from this digit (Figure 6j) shows that it was amputated at the proximal P3 transecting the os hole. By 7 weeks, skeletal staining of the regrown digit (Figure 6j) shows that the P3 bone was almost completely regrown. The regrown P3 shows trabecular appearance that is similar in general structure but not identical to the original P3. Another dramatic response was observed from another digit, which began reforming the nail by 7 weeks (Figure 6h). Skeletal staining of the portion removed from this digit shows that it was amputated across the P2 bone, removing the entire epiphyseal cap along with the sesamoid bone (Figure 6k). Skeletal staining 
455 of the regenerating digit shows that the epiphyseal cap was regrown, along with its associated 456 sesamoid bone. Moreover, articulating from the regenerated P2 appears to be the beginning of 457 the

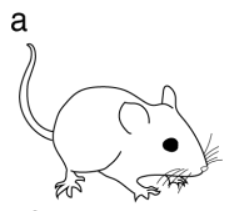

Amputate on hind paw

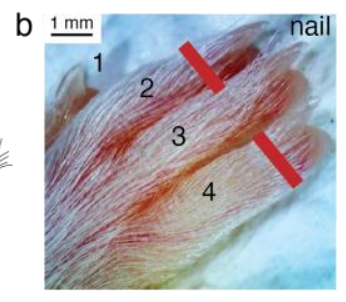

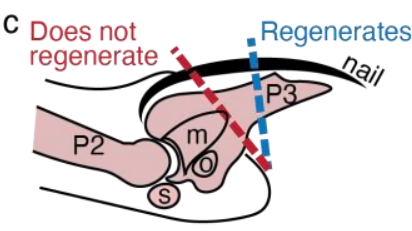

P2: mid phalange 0 : os hole P3: distal phalange s: sesamoid bone m: marrow cavity
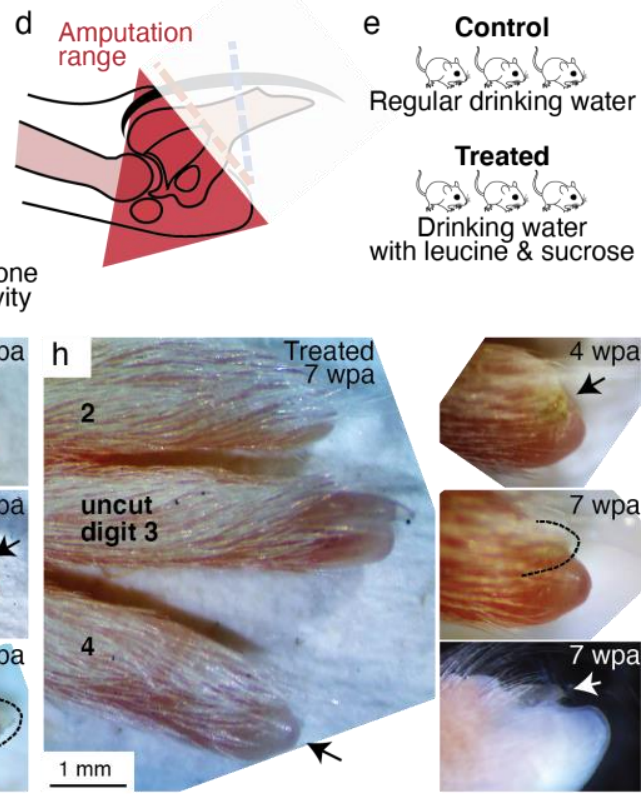
Control

\section{Treated}

ar: Drinking water with leucine \& sucrose
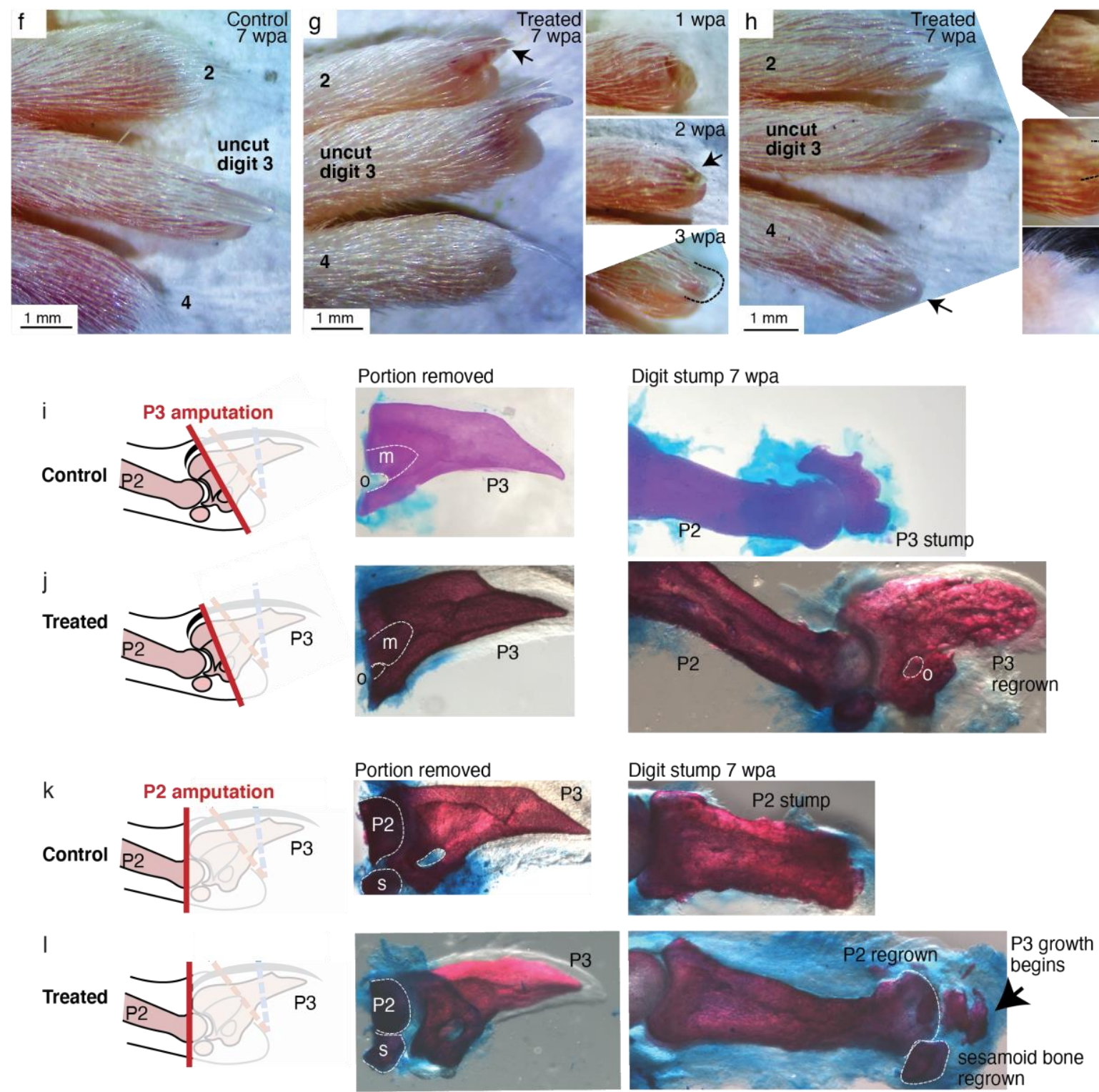
Figure 6. Leucine and sucrose induced regeneration in adult mouse digit.

(a-b) Amputation was performed on hindpaws of adult (3-6 month old) mice, on digits 2 and 4, proximal to the nail.

(c) Schematic of the distal phalange (P3) and middle phalange (P2). Amputations that remove $<30 \%$ of P3 (blue line) regenerate, whereas amputations that remove $>60 \%$ of P3 (red line) do not regenerate. Amputations in the intermediate region can occasionally show partial regenerative response.

(d) Amputations in this study were performed within the red-shaded triangle.

(e) Amputated mice were given regular drinking water (control) or drinking water supplemented with $1.5 \%$ L-leucine, $1.5 \%$ L-glutamine, and 4-10 w/v \% sucrose (2 exps with 4\%, 3 exps with 10\%). Drinking water, control and treated, was refreshed weekly.

(f) A representative paw from the control group. The amputated digits 2 and 4 simply healed the wound and did not regrow the distal phalange.

(g) In this treated mouse, digit 2 (arrow) regrew the distal phalange and nail. Insets on the right show the digit at earlier time points. At week 1, the amputation site still appeared inflamed. At week 3, the beginning of the nail appears (arrow). At week 3, a clear nail plate was observed.

(h) In this treated mouse, digit 4 (arrow) regrew and began to show nail reformation by week 4 (top inset, see arrow), that turns into a clear nail plate by week 7 (middle inset), as can be seen more clearly from the side-view darkfield image (bottom inset).

(i-l) Whole-mount skeletal staining. Dissected digits were stained with Alizarin red, an anionic dye that highly localizes to the bone. Left panels show illustration of the amputation plane, middle panels show skeletal staining of the portions removed, and right panels show skeletal staining of the digit stumps 7 weeks after amputation.

2 supplements: Figure 6-figure supplement 1-2. Source file: Mouse data.xls

next phalangeal bone (arrow, Figure 6k). To our knowledge, the regenerative response observed in these digits represents the most dramatic extent of self-organized mammalian digit regeneration reported thus far. Distal phalange regeneration in adults has not been reported, while interphalangeal joint formation from a P2 amputation has been achieved only through sequential Bmp administration ( $\mathrm{Yu}$ et al., 2019) and there has been no documentation of the regrowth of the sesamoid bone.

\section{Discussion}

In this study, amputations were performed on Aurelia appendage, Drosophila limb, and mouse digit. None of these animals are known to regenerate robustly (Aurelia) if at all (Drosophila and mouse) from these amputations. Upon administration of L-leucine and sugar/insulin, dramatic regenerative response was observed in all systems. The conserved effect of nutrient supplementation across three species that span more than 500 million years of evolutionary divergence suggests energetic parameters as ancestral regulators of regeneration activation in animals.

While we did not test the regenerative effect of hypoxia beyond Aurelia, it is notable that in mice hypoxia coaxes cardiomyocytes to re-enter cell cycle (Kimura et al., 2015) and activating HIF1 $\alpha$ promotes healing of ear hole punch injury (Zhang et al., 2015). Notably in Aurelia, the amputation bisected through the body, and more than appendage was in fact regenerated, e.g., circular muscle in the body is regrown (Figure 2e). Thus, nutrient supplementation may have regenerative effect in body parts beyond appendage.

The diverse physiologies of animals across phylogeny may seem difficult to reconcile with a conserved regulation of regeneration activation, especially in the view of regeneration as recapitulation of development. Growing a jellyfish appendage is different from building a fly leg 
or making a mouse digit. However, there is another way of looking at regeneration as a part of tissue plasticity (Galliot and Ghila, 2010). In this view of regeneration, upstream from tissuespecific morphogenesis is a conserved regulation of cell growth and proliferation. In support of this idea, early steps in regeneration across species and organs rely one way or another on proliferation by stem cells or differentiated cells re-entering cell cycle (Cox et al., 2019). We propose that in animals that poorly regenerate, high nutrient input turns on growth and anabolic states that promote tissue rebuilding upon injury.

That regenerative response can be induced seemingly blurs the boundary between regenerating versus non-regenerating animals, because the factors identified in the study are not exotic. Variations in amino acids, carbohydrates, and oxygen levels are conditions that the animals can plausibly encounter in nature. These observations highlight two potential insights into regeneration. First, regeneration is environmentally dependent. An animal would stop at wound healing under low-energy conditions and regenerate in energy-replete conditions. In this view, for the animals examined in this study, the typical laboratory conditions may simply not be conducive to regeneration. Alternatively, the interpretation we favor, what we observed is inherent regeneration, which can be activated with broad environmental factors. We favor this interpretation because the regenerative response was unusually variable. The variability stands in stark contrast to the robust regeneration in e.g., axolotl, planaria, or hydra. Whereas wild-type processes tend to be robust, mutations produce phenotypes that are sensitive to variations in physiological parameters. Thus, just like mutant phenotypes show varying penetrance and expressivity, the variable regenerative response speaks to us as a fundamental consequence of activating a latent biological module. In this interpretation, the ordinariness of the activators suggests ancestral regeneration as part of a response to broad environmental stimuli. It would be interesting next to identify individual differences that contribute to varying propensities to mount regenerative response.

In particular, the conserved effects of nutrient supplementation suggest that regeneration might have originally been a part of growth response to abundant environments. No nutrient dependence has been observed in highly regenerating animal models such as planaria, hydra, and axolotl. Environment-dependent plasticity, however, is pervasive in development, physiology, behavior, and phenology (West-Eberhard, 2003; Mockzek et al., 2011). We therefore conjecture that environment-dependent plasticity may have characterized the ancestral form of regeneration. In this conjecture, present regenerating lineages might have decoupled the linkage with environmental input and genetically assimilated regenerative response - because regeneration is adaptive or coupled to a strongly selected process, e.g., reproduction. In parallel, non- or poorly regenerating animals might have also weakened the linkage with environmental input, but to silence the regenerative response. This predicts an ancient form of a robustly regenerative animal (like planaria, hydra, axolotl) that tunes its regeneration frequency to nutrient abundance. Such plasticity has been reported in the basal lineage Ctenophora (Bading et al., 2017).

In conclusion, this study suggests that an inherent ability for appendage regeneration is retained in non-regenerating animals and can be unlocked with a conserved strategy. The treatments across species were not exactly identical, and correspondingly there might be differences in the precise molecular mechanisms - in spite of which they could be applied across species in a predictive manner. In line with our findings, the role of nutrients in promoting regeneration was reported in yet another species (in the Xenopus tadpoles, Williams et al., 2021). While the observed regenerative response is not perfect, this motivates further investigation into potentially more promoting factors or the possibility of combining broad promoting factors with 
553 species- or tissue-specific morphogenetic regulators. Reiterating Spallanzani's hope, Marcus 554 Singer supposed half a century ago that "... every organ has the power to regrow lying latent 555 within it, needing only the appropriate 'useful dispositions' to bring it out (Singer, 1958)." The 556 surprise, in hindsight, is the simplicity by which the regenerative state can be promoted with ad 557 libitum amino acid and sugar supplementation. This simplicity demonstrates a much broader 558 possibility of organismal regeneration, and can help accelerate progress in regeneration induction 559 across animals. 


\section{Acknowledgements}

561 The authors thank Kiersten Darrow and Michael Schaadt at the Cabrillo Marine Aquarium,

562 Cabrillo, CA for the gifts of Aurelia polyps and help with jellyfish culture, Patrick Leahy at the

563 Caltech's Kerckhoff Marine Laboratory, Corona Del Mar, CA for the help with setting up

564 Aurelia experiments in natural habitat, Matthew Hunt from the Kavli Nanoscience Institute at

565 Caltech for help with SEM imaging, James McGehee, Angela Stathopolous, Peter Lee, Kai Zinn

566 for sharing Drosophila strains, Yujing Yang and Long Cai for help with imaging, and Carlos

567 Lois for demonstration of digit amputation. We thank Gertrud Schupbach, Natalie Andrew, and

568 Aki Ohdera for comments on the manuscript; and Katalina Fejes-Toth, Luis del Peso, Aditya

569 Saxena, and Marta Truchado Garcia for discussion. This work was supported by the National

570 Science Foundation Graduate Research Fellowship Program (1144469; to M.J.A.), the James E.

571 and Charlotte Fedde Cordes Postdoctoral Fellowship in Biology (to D.A.G.); the James S.

572 McDonnell Foundation for Complex Systems Science (220020365; to L.G.), the Center for

573 Environmental Microbial Interactions at Caltech (to Z.C. and L.G.), the Center of Evolutionary

574 Sciences at Caltech (to Y.L. and L.G.), the Summer Undergraduate Research Fellowships (to I.L.

575 and L.G.), and Charles Trimble and Caltech's Biology and Biological Engineering Chair's

576 Council Inducing Regeneration Fund (to L.G.).

\section{Methods}

578 Key Resource Table

\begin{tabular}{|c|c|c|c|c|}
\hline $\begin{array}{l}\text { Reagent type } \\
\text { (species) or } \\
\text { resource }\end{array}$ & Designation & $\begin{array}{l}\text { Source or } \\
\text { reference }\end{array}$ & Identifiers & $\begin{array}{l}\text { Additional } \\
\text { information }\end{array}$ \\
\hline $\begin{array}{l}\text { Strain, strain } \\
\text { background (Aurelia } \\
\text { aurita) }\end{array}$ & $\underset{1}{\text { Aurelia aurita sp. }}$ & $\begin{array}{l}\text { Gift from the } \\
\text { Cabrillo Marine } \\
\text { Aquarium,San } \\
\text { Pedro, CA }\end{array}$ & & $\begin{array}{l}\text { Alternatively } \\
\text { named Aurelia } \\
\text { coerulea } \\
\text { (Scorrano et al., } \\
\text { 2016). }\end{array}$ \\
\hline $\begin{array}{l}\text { Strain, strain } \\
\text { background } \\
\text { (Drosophila } \\
\text { melanogaster) }\end{array}$ & OregonR & $\begin{array}{l}\text { Gift from Angela } \\
\text { Stathopolous' } \\
\text { lab, Caltech }\end{array}$ & & RRID:BDSC_5 \\
\hline $\begin{array}{l}\text { Strain, strain } \\
\text { background } \\
\text { (Drosophila } \\
\text { melanogaster) }\end{array}$ & Cantons & $\begin{array}{l}\text { Gift from Kai } \\
\text { Zinn lab, } \\
\text { Caltech }\end{array}$ & & $\begin{array}{l}\text { RRID:BDSC_6 } \\
4349\end{array}$ \\
\hline $\begin{array}{l}\text { Strain, strain } \\
\text { background (Mus } \\
\text { musculus) }\end{array}$ & CD1 & $\begin{array}{l}\text { Charles River } \\
\text { Laboratories }\end{array}$ & Strain 022 & $\begin{array}{l}\text { Female, } 3-6 \\
\text { months old } \\
\text { RRID:IMSR_C } \\
\text { RL:022 }\end{array}$ \\
\hline $\begin{array}{l}\text { Chemical compound, } \\
\text { drug }\end{array}$ & L-leucine & Sigma & L1002 & $\begin{array}{l}\text { Methyl-ester } \\
\text { hydrochloride }\end{array}$ \\
\hline
\end{tabular}




\begin{tabular}{|c|c|c|c|c|}
\hline $\begin{array}{l}\text { Chemical compound, } \\
\text { drug }\end{array}$ & L-leucine & Sigma & L8000 & \\
\hline $\begin{array}{l}\text { Chemical compound, } \\
\text { drug }\end{array}$ & L-leucine & VWR & E811 & USP grade \\
\hline $\begin{array}{l}\text { Chemical compound, } \\
\text { drug }\end{array}$ & L-glutamine & Sigma & G3126 & \\
\hline $\begin{array}{l}\text { Chemical compound, } \\
\text { drug }\end{array}$ & L-glutamine & Sigma & G8540 & USP grade \\
\hline $\begin{array}{l}\text { Chemical compound, } \\
\text { drug }\end{array}$ & Sucrose & Avantor & 8360 & AR ACS grade \\
\hline $\begin{array}{l}\text { Peptide, recombinant } \\
\text { protein }\end{array}$ & Insulin & Sigma & 10908 & \\
\hline $\begin{array}{l}\text { Peptide, recombinant } \\
\text { protein }\end{array}$ & Insulin & MP Biomedicals & 0219390080 & \\
\hline Antibody & $\begin{array}{l}\text { anti-tyrosinated } \\
\text { alpha tubulin (Rat } \\
\text { monoclonal) }\end{array}$ & Sigma & MAB1864-I & $\begin{array}{l}(1: 200) \\
\text { RRID:AB_289 } \\
4901\end{array}$ \\
\hline Antibody & $\begin{array}{l}\text { Goat anti-mouse } \\
\text { Alexa Fluor } 488 \\
\text { (Goat polyclonal) }\end{array}$ & $\begin{array}{l}\text { Life } \\
\text { Technologies } \\
\text { (Thermo Fisher) }\end{array}$ & A11029 & $\begin{array}{l}(1: 200) \\
\text { RRID:AB_289 } \\
4900\end{array}$ \\
\hline Other & $\begin{array}{l}\text { Alexa Fluor } 555 \\
\text { phalloidin }\end{array}$ & $\begin{array}{l}\text { Life } \\
\text { Technologies } \\
\text { (Thermo Fisher) }\end{array}$ & A12379 & $\begin{array}{l}\text { Histological } \\
\text { stains, (1:20) }\end{array}$ \\
\hline Other & Hoechst 33342 & Sigma & B2261 & $\begin{array}{l}\text { Histological } \\
\text { stains, (1:10) }\end{array}$ \\
\hline Other & $\begin{array}{l}\text { Vectashield } \\
\text { mounting medium } \\
\text { with DAPI }\end{array}$ & Vector & $\mathrm{H} 1200$ & $\begin{array}{l}\text { Histological } \\
\text { stains, }(1: 1)\end{array}$ \\
\hline Other & Alizarin red & $\begin{array}{l}\text { Beantown } \\
\text { Chemical }\end{array}$ & BT144735 & $\begin{array}{l}\text { Histological } \\
\text { stains, } 0.005 \%\end{array}$ \\
\hline Other & Alcian blue & Across Organics & $\begin{array}{l}\text { AC40046- } \\
0100\end{array}$ & $\begin{array}{l}\text { Histological } \\
\text { stains, } 0.015 \%\end{array}$ \\
\hline
\end{tabular}

581 The experiments were performed in Aurelia aurita sp. 1 strain, also alternatively named Aurelia 582 coerulea based on recent molecular classification (Scorrano et al., 2016). Polyps were reared at 
$68^{\circ} \mathrm{F}$, in $32 \mathrm{ppt}$ artificial seawater (ASW, Instant Ocean), and fed daily with brine shrimps (Artemia nauplii) enriched with Nannochloropsis algae (both from Brine Shrimp Direct). To induce strobilation, polyps were incubated in 25 M 5-methoxy-2-methyl-indole (Sigma M15451) at $68^{\circ} \mathrm{F}$ for an hour (Fuchs et. al, 2014). Ephyrae typically began to strobilate within a week. Strobilated ephyrae were fed daily with high amount of rotifers (Brachionus plicatilis, Reed Mariculture) until amputation time.

Experiment in the original habitat. The polyp population in the study arose from parental polyps collected off the coast of Long Beach, CA $\left(33^{\circ} 46^{\prime} 04.2^{\prime \prime} \mathrm{N} 118^{\circ} 07^{\prime} 44.2^{\prime \prime} \mathrm{W}\right.$, GPS: 33.7678376,-118.1289559). Ephyrae were amputated in location and immediately after submersed in the ocean. For submerging the amputated ephyrae in the ocean, a two-layered aquarium was custom-built. Ephyrae were placed in plastic canisters with a $7 \mathrm{~cm}$ diameter hole cut in the lid and covered with a $250 \mu \mathrm{m}$ plastic screen. The canisters were then placed in a thick plastic tank fitted with a $500 \mu \mathrm{m}$ plastic screen on top. This design offers protection to the ephyrae against predators and strong waves, while at the same time allowing exchange of water, zooplanktons, and other particulates. Ephyrae were collected after two weeks.

Regeneration experiments. All experiments were performed at $68^{\circ} \mathrm{F}$. 2-3 days old ephyrae were anesthetized in $400 \mu \mathrm{M}$ menthol and amputated using a razor blade mounted on an $\mathrm{x}$-acto knife handle. Amputated ephyrae were let to recover in $1 \mathrm{~L}$ sand settling cones (Nalgene Imhoff, Figure 3-figure supplement 1). In each experiment, 90 animals were amputated for each condition (e.g., 90 animals for control and 90 animals for treated). Because of the varying baseline across strobilation batches, each experiment was repeated across 2-5 strobilation batches (biological replicates). These sample sizes were chosen to obtain a 95\% confidence level on the treatment effect (statistical analysis described below). Hundreds of experimental animals were first amputated, mixed together in a beaker, and then randomly allocated to the control or treatment groups. Regeneration was assessed at various times for 1-2 weeks after amputation, before onset of maturation to medusa. All data were included in the analysis.

Rationale for the amputation scheme. Among the possible amputation schemes, 3-arm amputation was chosen because it could be performed fastest. Removing 1 arm requires carefully cutting across the base of the arm while avoiding injuring the surrounding body. Removing 2 arms is less hard but still requires awkward positioning of the knife. Removing 4 arms again takes more time because it requires cutting through the large protruding manubrium, which also affects the animal's feeding ability. The fast 3-arm amputation facilitates testing hundreds of ephyrae per experiment.

Nutrients. Amputated ephyrae were fed daily with rotifers. The number of rotifers was estimated using a 6-well plate fitted with STEMgrid ${ }^{\mathrm{TM}}$ (the same principle as using a hemocytometer). In this study, low food was $\sim 10-20$ rotifers/ephyra and high food was $\sim 40$ rotifers/ephyra. To replicate the study, these numbers should only be used as initial estimates, as what is "low" or "high" food amount may easily vary across lab cultures (e.g., rotifer culture, differences across Aurelia strains, etc.). Most if not all rotifers were typically consumed within an hour (determined by measuring the rotifers in the water).

Insulin. Immediately after amputation, ephyrae were placed in ASW supplemented with $500 \mathrm{nM}$ human recombinant insulin (Sigma I0908). Insulin was refreshed weekly. To determine the concentration used, a range of concentrations, $10 \mathrm{nM}$ to $3 \mathrm{mM}$, were tested. The concentration $500 \mathrm{nM}$ was chosen as it maximized regeneration frequency while avoiding solubility problems. 
627 To control that the effect of insulin was not due to non-specific additions of proteins, BSA at 500 $628 \mathrm{nM}$ was tested.

629 Hypoxia. Immediately after amputation, ephyrae were placed in hypoxic ASW. To create a 630 hypoxic environment, nitrogen or argon, instead of ambient air, was pumped into the bubbler 631 cone, beginning from the day before the experiment and maintained throughout the duration of 632 the experiment. The bubbler cone was sealed with parafilm to maintain the lowered oxygen 633 level. The nitrogen/argon flow was adjusted to achieve 50\% reduction in the dissolved oxygen 634 level. Dissolved oxygen level was measured using a Clark-type electrode Unisense OX-500 635 microsensor. The measurement was normalized to oxygen level in control ASW bubbled 636 normally with ambient air. Oxygen measurement was performed prior to the experiment and 637 subsequently every 3 days.

638 L-leucine. Immediately after amputation, ephyrae were placed in ASW supplemented with 100 $639 \mu \mathrm{M}$ L-leucine (Sigma L1002, the cell-permeable methyl ester hydrochloride form). L-leucine 640 was refreshed weekly. To determine the concentration used, a range of concentrations from one 641 to hundreds of $\mathrm{mM}$ was tested. The concentration of $100 \mathrm{mM}$ was chosen as it maximized the 642 regeneration frequency without non-specific, negative effects.

643 Statistical analysis. To assess the statistical significance of the treatments, meta-analysis of 644 effect size was performed (Borenstein et al., 2009). The effect size metrics used are determined 645 by the form of the dataset. For measurements of frequencies (e.g., regeneration frequency), the 646 datasets are in the form of a 2 x 2 table of dichotomous variables,

\begin{tabular}{|l|c|c|}
\hline & \# ephyrae that regenerate & \# ephyrae that do not regenerate \\
\hline Control & $\mathrm{a}$ & $\mathrm{b}$ \\
\hline Treatment & $\mathrm{c}$ & $\mathrm{d}$ \\
\hline
\end{tabular}

647 For such $2 \times 2$ datasets, in situations where the baseline varies (e.g., varying baseline 648 regeneration across clutches), the commonly used measures of effect size are the Risk Ratio 649 (RR),

$$
\mathrm{RR}=\frac{\left(\frac{\# \text { ephyrae that regenerate }}{\text { total \# ephyrae }}\right) \text { in treated group }}{\left(\frac{\# \text { ephyrae that regenerate }}{\text { total \# ephyrae }}\right) \text { in control group }}=\frac{\frac{\mathrm{c}}{(\mathrm{c}+\mathrm{d})}}{\frac{\mathrm{a}}{(\mathrm{a}+\mathrm{b})}}
$$

650 and the Odds Ratio (OR),

$$
\mathrm{OR}=\frac{\left(\frac{\# \text { ephyrae that regenerate }}{\# \text { ephyrae that do not regenerate }}\right) \text { in treated group }}{\left(\frac{\# \text { ephyrae that regenerate }}{\# \text { ephyrae that do not regenerate }}\right) \text { in control group }}=\frac{\frac{\mathrm{c}}{(\mathrm{c}+\mathrm{d})}}{\frac{\mathrm{a}}{(\mathrm{a}+\mathrm{b})}}
$$

651 RR compares the probability of an outcome in treated vs control group, whereas OR compares 652 the odds of an outcome in treated vs control group.

653 For measurements of arm length and body size, the datasets are in the form of continuous 654 variables. For such data, the commonly used effect size is the Response Ratio (R),

$$
\mathrm{R}=\frac{\text { mean arm length in treated group }}{\text { mean arm length in control group }}
$$


$655 \mathrm{R}$ evaluates the proportionate change that results from a treatment, and is the meaningful effect 656 size to use when the outcome of a treatment is measured on a physical scale, e.g., length or area 657 (as opposed to arbitrary scale, e.g., happiness level). Experiments where regeneration in one of 658 the groups occurred in 0 ephyra were necessarily excluded.

Having computed the effect size (RR, OR, or R) within each experiment, meta-analysis of 660 the effect size across experiments was performed. The metafor package (Viechtbauer, 2010) in R 661 was used, with fixed-effect model (for nutrients and leucine) or random-effect restricted 662 maximum likelihood model (for insulin and hypoxia, which had different control conditions 663 across the experiments). Statistical coefficients were based on normal distribution.

664 Phalloidin and tyrosinated tubulin staining. All steps were performed at room temperature, 665 unless indicated otherwise. Ephyrae were first anesthetized in $400 \mu \mathrm{M}$ menthol, which minimizes 666 curling during fixing. Next, ephyrae were fixed in 3.7\% (v/v) formaldehyde (in PBS) for 15 667 minutes, permeabilized in 0.5\% Triton X-100 (in PBS) for 5 minutes, and blocked in 3\% (w/v) 668 BSA for 2 minutes. For neuron staining, ephyrae were incubated in 1:200 mouse anti-tyrosinated 669 alpha tubulin antibody (Sigma MAB1864-I) overnight at $4^{\circ} \mathrm{C}$, and then in 1:200 goat-anti-mouse 670 Alexa Fluor 488 (Life Technologies A11029) overnight in the dark at $4^{\circ} \mathrm{C}$. Primary or secondary 671 antibodies were diluted in 3\% BSA. For actin staining, ephyrae were incubated in 1:20 Alexa 672 Fluor 555 Phalloidin (Life Technologies A12379) overnight or for 2 hours in the dark at $4^{\circ} \mathrm{C}$. For 673 nuclei staining, ephyrae were incubated in 1:10 Hoechst 33342 (Sigma B2261) for 30 minutes in 674 the dark.

675 Microscopy._Ephyrae were imaged anesthetized in menthol._Brightfield images, fluorescent 676 images, and movies were taken with the Zeiss AxioZoom.V16 stereo zoom microscope and 677 AxioCam HR 13-megapixel camera. Optical sectioning was performed with ApoTome.2.

\section{Drosophila melanogaster}

679 CantonS wild-type strain was a gift from Peter Lee in Kai Zinn's lab at Caltech. OregonR was a 680 gift from James McGehee in Angela Stathopolous' lab at Caltech. OregonR and CantonS flies 681 were reared under standard conditions at $23^{\circ} \mathrm{C}$, sometime supplemented with baker's yeast.

682 Regeneration experiments. Amputation was performed on adult flies 2-7 days after eclosion. 683 Flies were anesthetized with $\mathrm{CO}_{2}$, placed under a dissection microscope, and tibia amputated 684 using a spring scissors (Fine Science Tools, 91500-09) and superfine dissecting forceps (VWR, 685 82027-402). See Figure 4 for detailed description of the amputation plane. Recovering Drosophila were allocated randomly to vials with standard lab fly food (control) or standard lab fly food mixed with $5 \mathrm{mM}$ L-Leucine (Sigma L8000), $5 \mathrm{mM}$ L-Glutamine (Sigma G3126), and $0.1 \mathrm{mg} / \mathrm{mL}$ insulin (human recombinant, MP Biomedicals 0219390080). To introduce the nutrient supplements, the fly food was microwaved in short pulses, such that the topmost layer of the food was liquified. The supplements in aqueous stocks were then pipetted into this liquified layer. Food was allowed to re-set at $4^{\circ} \mathrm{C}$ for at least 20 minutes. New food was prepared fresh every 2 days, and flies were moved into freshly prepared treated food every 2 days, throughout the course of the 2- to 3-week experiment.

694 Single-fly tracking. Amputation and treatment were performed as in the bulk regeneration 695 experiments described above, with the following modifications. Canton S flies were amputated 696 in different limbs, and were housed in small groups such that in any given vial, each fly was 697 uniquely identifiable by sex and amputated limb. Typically, 1-6 flies are housed in each vial. 3- 
5-day treatment produced similar response as sustained treatment; therefore, for simplicity, treatment was performed for 3-5 days. Treated flies, but not control flies, were sometime continued on yeast supplementation. Flies were imaged immediately after amputation and 1-3 additional times over the course of 2-4 weeks. As anesthetized flies jitter, images were taken in a video format, and single frames were then selected for analysis in which the leg stump was in focus. Tibia length was quantified in Image $J$ as the diameter of the minimum enclosing circle of the leg to achieve rotation-invariant assessments. Identical imaging and analysis procedures were used for treated and control flies.

Statistical analysis and blind. Blind measurements were performed on one pair of control and treated datasets. Statistical comparison of percent change in length in control and treated leg stumps was performed using the non-parametric Kruskal-Wallis test. Samples were assigned integer ranks from smallest to largest by quantity, and then differences between conditions were assessed with respect to the ranks. The p-value tests the null hypothesis that the data are drawn from the same distribution.

DAPI staining. Fly tibias were dissected and washed in $70 \%$ ethanol $(<1 \mathrm{~min})$ to decrease the hydrophobicity of the cuticle and washed in PBS with $0.3 \%$ Triton-X for 10 minutes. The legs were fixed in $4 \%$ paraformaldehyde (in PBS) overnight at $4^{\circ} \mathrm{C}$ and washed five times for 20 minutes each in PBS with $0.3 \%$ Triton-X. The legs were equilibrated in Vectashield mounting medium with DAPI (Vector H-1200) overnight at $4{ }^{\circ} \mathrm{C}$, and imaged using Zeiss AxioZoom.V16 stereo zoom microscope with AxioCam HR 13-megapixel camera. Confocal imaging was performed using X-Light V2 spinning disk mounted on the Olympus IX81 inverted microscope.

Live fly imaging. Flies anesthetized on a $\mathrm{CO}_{2}$ bed were imaged under a Zeiss SteREO Discovery.V8 stereomicroscope equipped with the Zeiss AxioCam 503 color camera.

Electron microscopy. Environmental scanning electron microscopy (ESEM) was performed on a FEI Quanta 200F (FEI, Hillsboro, Oregon). Whole live flies were mounted onto the SEM stub with copper tape. ESEM images were attained at a pressure of 0.1 mbar and $5 \mathrm{kV}$ at a working distance of 9-12 mm, with water as the ionizing gas.

\section{Mus musculus}

All studies comply with relevant ethical regulations for animal testing and research, and received ethical approval by the Institutional Animal Care and Use Committees at the California Institute of Technology.

Strain. Adult female (3-6 months old) wild-type CD1 mice (Charles River Laboratories strain 022) were used for all regeneration studies.

Regeneration experiments. Digit amputation was performed following the established protocol in the field (Simkin et al., 2013). Mice were anesthetized with 1-5\% isoflurane (in oxygen) in an induction chamber, followed by maintenance on a nosecone. The mouse was positioned on its belly with its hind paws outstretched and the ventral side of the paw facing upwards. SustainedRelease Buprenorphine was administered (Buprenorphine SR LAB®) at $0.5 \mathrm{mg} / \mathrm{kg}$ subcutaneously as an analgesic. Blood flow to the hindlimb was stemmed by tying a rubber band around the ankle and clamping it with a hemostat. All surgical procedures were carried out under a Zeiss Stemi 305 dissection microscope. An initial incision, parallel to the position of foot, was made through the ventral fat pad using Vannas spring scissors (World Precision Instruments, 14003). The length of this incision was determined by the amount of ventral skin needed to seal 
the digit amputation wound completely. The ventral skin freed in the initial incision was peeled back using surgical forceps, and a no. 10 scalpel (Sklar, 06-3110) was used to amputate and bisect the digit completely through the second or third phalange. Digits 2 and 4 on the right hind paw were operated on in this fashion, while digit 3 remained unamputated as an internal control. The amputation wound was immediately closed with the ventral skin flap and sealed with GLUture (Zoetis, Kalamazoo, MI). Amputated portions were immediately fixed as control for skeletal staining. Dissolved 1.5\% L-leucine (USP grade, VWR E811), 1.5\% L-glutamine (USP grade, Sigma G8540), and 4-10\% sucrose (AR ACS grade, Avantor 8360) in drinking water was administered to mice in the experimental group ad libitum after amputation. Control mice were given untreated drinking water. Drinking water was refreshed weekly for both control and experimental groups, and treated water was made fresh on the day that drinking water was replaced. The amputated digit stumps were photographed weekly for 7-8 weeks, at which time the digits were dissected for skeletal staining.

Statistical analysis. The sample size in the experiment balanced the aim of achieving $>90 \%$ confidence level with ethical consideration of minimizing the number of animals used. Animals were randomly allocated to the control or treatment group. No restricted randomization was applied. For weight measurement, the unit of analysis is a single animal. For regeneration phenotype, the unit of analysis is a single digit. Student's t-test was used to evaluate the null hypothesis that there is no difference between the control and treated groups. $95 \%$ confidence intervals were computed assuming normal distribution. All data were included in the analysis.

Mouse digit dissection and skeletal staining. Mice were euthanized and digits 2, 3 and 4 were removed with a no. 10 scalpel (Sklar, 06-3110) through the first phalange. Excess skin and flesh were removed with spring scissors (Fine Science Tools, 91500-09) and fine dissecting forceps (Fine Science Tools, 11254-20). All digits analyzed by whole-mount skeletal stains were prepared with a standard alizarin red and alcian blue staining protocol (Mcleod, 1980) Digits were dehydrated in $95 \%$ ethanol for 1 day, and incubated in staining solution $(0.005 \%$ alizarin red (Beantown Chemical, BT144735), 0.015\% alcian blue (Acros Organics, AC40046-0100), $5 \%$ acetic acid, $60 \%$ ethanol) for 1 day at $37^{\circ} \mathrm{C}$. Tissue was cleared in $2 \%$ potassium hydroxide at room temperature for 1 day, $1 \%$ potassium hydroxide for 1 day, and then taken through an increasing glycerol series $(25 \%, 50 \%, 75 \%, 100 \%)$. The stained samples were imaged on Zeiss AxioZoom.V16 stereo zoom microscope with a Zeiss AxioCam 503 color camera or a Zeiss Stemi 305 dissection microscope with an iPhone 6 camera.

\section{Data Availability}

Raw image data of ephyrae analyzed in Figure 3, raw video data of flies analyzed in Figure 5, and image data of mouse digits analyzed in Figure 6 are deposited in the open repository CaltechDATA.

Aurelia data: https://doi.org/10.22002/D1.2076

Drosophila data: https://doi.org/10.22002/D1.2157

Mouse data: https://doi.org/10.22002/D1.1790

\section{List of source files, codes, and videos}

Video 1. Arm regenerates pulse synchronously with existing arms. This video was taken 2 weeks after amputation. The synchronous pulsing suggests functional rebuilding of neuromuscular tissues. 
784 Viedo 2. Experimental setup in Aurelia. See Figure 3-figure supplement 1 for more details.

785 Aurelia screen.xls. Figure 3-Source data. This spreadsheet details the factors screened in

786 Aurelia, rationale for screening the factors, targets of the molecular modulators tested, doses or

787 parameters tested, estimate number of ephyrae tested, and relevant references.

788 Aurelia data.xls. Figure 3-Source data. This spreadsheet contains the raw data of Figure 3 789 and its figure supplements.

790 R-codes.rtf. Figure 3-Source code. This R-codes compute the statistics of Figure 3 and its 791 figure supplements.

792 Drosophila data.xls. Figure 5-Source data. This spreadsheet contains the raw data of Figure 7935 and its figure supplements.

794 DrosophilaCode.m. Figure 5-Source code. This Matlab code plots Figure 5 and its figure 795 supplements.

796 Mouse data.xls. Figure 6-Source data. This spreadsheet contains the raw data of the mouse 797 digit phenotype in Figure 6 and its figure supplements. The spreadsheet documents the raw 798 image filename, amputation type, and phenotype scoring.

799 References

800 Abrams, M. J., \& Goentoro, L. (2016). Symmetrization in jellyfish: Reorganization to regain 801 function, and not lost parts. Zoology, 119(1), 1-3. https://doi.org/10.1016/j.zool.2015.10.001

802 Alvarado, A. S. (2000). Regeneration in the metazoans: Why does it happen? BioEssays, 22(6), 803 578-590. https://doi.org/10.1002/(SICI)1521-1878(200006)22:6<578::AID-BIES11>3.0.CO;2$804 \quad \% 23$

805 Bading, K. T., Kaehlert, S., Chi, X., Jaspers, C., Martindale, M. Q., \& Javidpour, J. (2017). Food 806 availability drives plastic self-repair response in a basal metazoan- case study on the ctenophore 807 Mnemiopsis leidyi A. Agassiz 1865. Scientific Reports, 7(1). https://doi.org/10.1038/s41598-017$808 \quad \underline{16346-\mathrm{w}}$

809 Bely, A. E., \& Nyberg, K. G. (2010). Evolution of animal regeneration: Re-emergence of a field. 810 Trends in Ecology \& Evolution, 25(3), 161-170. https://doi.org/10.1016/j.tree.2009.08.005

811 Borenstein, M., Hedges, L., V., Higgins, J., P. T., \& Rothstein, H. R. (2009). Introduction to 812 Meta-Analysis. John Wiley \& Sons, 813 https://onlinelibrary.wiley.com/doi/pdf/10.1002/9780470743386

814 Cao, D., Lu, H., Lewis, T. L., \& Li, L. (2007). Intake of Sucrose-sweetened Water Induces 815 Insulin Resistance and Exacerbates Memory Deficits and Amyloidosis in a Transgenic Mouse 816 Model of Alzheimer Disease. Journal of Biological Chemistry, 282(50), 36275-36282. 817 https://doi.org/10.1074/jbc.M703561200

818 Carlson, B., M. (2007). Principles of Regenerative Biology. Elsevier.

819 Chamberlain, C. S., Jeffery, J. J., Leiferman, E. M., Yildirim, T., Sun, X., Baer, G. S., Murphy, 820 W. L., \& Vanderby, R. (2017). Level-Specific Amputations and Resulting Regenerative 821 Outcomes in the Mouse Distal Phalanx. Wound Repair and Regeneration: Official Publication 822 of the Wound Healing Society [and] the European Tissue Repair Society, 25(3), 443-453. 823 https://doi.org/10.1111/wrr.12544 
824 Cox, B. D., Yun, M. H., \& Poss, K. D. (2019). Can laboratory model systems instruct human 825 limb regeneration? Development, 146(20), dev181016. https://doi.org/10.1242/dev.181016

826 Endo, T., Bryant, S. V., \& Gardiner, D. M. (2004). A stepwise model system for limb regeneration. Developmental Biology, 11. https://10.1016/j.ydbio.2004.02.016

828 Eyal, S., Rubin, S., Krief, S., Levin, L., \& Zelzer, E. (2019). Common cellular origin and 829 diverging developmental programs for different sesamoid bones. Development, 146(4), 830 dev167452. https://doi.org/10.1242/dev.167452

831 Fuchs, B., Wang, W., Graspeuntner, S., Li, Y., Insua, S., Herbst, E. M., Dirksen, P., Böhm, A. M., Hemmrich, G., Sommer, F., Domazet-Lošo, T., Klostermeier, U. C., Anton-Erxleben, F., Rosenstiel, P., Bosch, T. C., \& Khalturin, K. (2014). Regulation of polyp-to-jellyfish transition in Aurelia aurita. Current

biology:

$C B, 24(3)$,

263-273.

https://doi.org/10.1016/j.cub.2013.12.003

836 Galliot, B., \& Ghila, L. (2010). Cell plasticity in homeostasis and regeneration. Molecular 837 Reproduction and Development, 77(10), 837-855. https://doi.org/10.1002/mrd.21206

838 Goss, R. J. (1992). The evolution of regeneration: Adaptive or inherent? Journal of Theoretical Biology, 159(2), 241-260. https://doi.org/10.1016/S0022-5193(05)80704-0

840 Graham, W. M. (2001). Size-based Prey Selectivity and Dietary Shifts in the Jellyfish, Aurelia 841

842 aurita. Journal of Plankton Research, 23(1), 67-74. https://doi.org/10.1093/plankt/23.1.67

Haddad, G. G., Sun, Y.-A., Wyman, R. J., \& Xu, T. (1997). Genetic basis of tolerance to O2 deprivation in. Proc. Natl. Acad. Sci. USA, 4.

Hariharan, I. K., Wake, D. B., \& Wake, M. H. (2016). Indeterminate Growth: Could It Represent the Ancestral Condition? Cold Spring Harbor Perspectives in Biology, 8(2). https://doi.org/10.1101/cshperspect.a019174

847 Herrera-Rincon, C., Golding, A. S., Moran, K. M., Harrison, C., Martyniuk, C. J., Guay, J. A., Zaltsman, J., Carabello, H., Kaplan, D. L., \& Levin, M. (2018). Brief Local Application of Progesterone via a Wearable Bioreactor Induces Long-Term Regenerative Response in Adult Xenopus Hindlimb. Cell Reports, 25(6), 1593-1609.e7. 851 https://doi.org/10.1016/j.celrep.2018.10.010

852 Hirose, K., Shiomi, T., Hozumi, S., \& Kikuchi, Y. (2014). Mechanistic target of rapamycin 853 complex 1 signaling regulates cell proliferation, cell survival, and differentiation in regenerating 854 zebrafish fins. BMC Developmental Biology, 14(1). https://doi.org/10.1186/s12861-014-0042-9

855 Hopkins, P., M., \& Das, S. (2015). Regeneration in Crustaceans. In E. S. Chang \& M. Thiel 856 (Eds.), The Natural History of the Crustacea (Vol. 4). Oxford University Press.

857 Illingworth, C., M. (1974). Trapped fingers and amputated finger tips in children. Journal of 858 Pediatric Surgery, 9(6), 853-858.

859 Kawakami, Y., Rodriguez Esteban, C., Raya, M., Kawakami, H., Martí, M., Dubova, I., \& 860 Izpisúa Belmonte, J. C. (2006). Wnt/ $\beta$-catenin signaling regulates vertebrate limb regeneration. 861 Genes \& Development, 20(23), 3232-3237. https://doi.org/10.1101/gad.1475106

862 Kimura, W., Xiao, F., Canseco, D. C., Muralidhar, S., Thet, S., Zhang, H. M., Abderrahman, Y., 863 Chen, R., Garcia, J. A., Shelton, J. M., Richardson, J. A., Ashour, A. M., Asaithamby, A., Liang, 
864 H., Xing, C., Lu, Z., Zhang, C. C., \& Sadek, H. A. (2015). Hypoxia fate mapping identifies

865 cycling cardiomyocytes in the adult heart. Nature, 523(7559), 226-230.

866 https://doi.org/10.1038/nature14582

867 Lai, A. G. \& Aboobaker, A. A. (2018) EvoRegen in animals: Time to uncover deep 868 conservatation or convergence of adult stem cell evolution and regenerative processes.

869 Developmental Biology, 433(2), 118-131. https://pubmed.ncbi.nlm.nih.gov/29198565/

870 Lehoczky, J. A., Robert, B., \& Tabin, C. J. (2011). Mouse digit tip regeneration is mediated by 871 fate-restricted progenitor cells. Proceedings of the National Academy of Sciences, 108(51), 872 20609-20614.https://doi.org/10.1073/pnas.1118017108

873 Lin, G., Chen, Y., \& Slack, J. M. W. (2013). Imparting regenerative capacity to limbs by 874 progenitor cell transplantation. Developmental Cell, 24(1), 41-51. 875 https://doi.org/10.1016/j.devcel.2012.11.017

876 Lucas, C. H. (2001). Reproduction and life history strategies of the common jellyfish, Aurelia aurita, in relation to its ambient environment. In J. E. Purcell, W. M. Graham, \& H. J. Dumont (Eds.), Jellyfish Blooms: Ecological and Societal Importance (pp. 229-246). Springer Netherlands. https://doi.org/10.1007/978-94-010-0722-1_19

880 Lynch, C. J., \& Adams, S. H. (2014). Branched-chain amino acids in metabolic signalling and 881 insulin resistance. Nature Reviews. Endocrinology, 10(12), $723-736$. 882 https://doi.org/10.1038/nrendo.2014.171

883 McLeod MJ (1980) Differential staining of cartilage and bone in whole mouse fetuses 884 by alcian blue and alizarin red S. Teratology 22:299e301.

885 Malandraki-Miller, S., Lopez, C. A., Al-Siddiqi, H., \& Carr, C. A. (2018). Changing Metabolism 886 in Differentiating Cardiac Progenitor Cells - Can Stem Cells Become Metabolically Flexible 887 Cardiomyocytes? Frontiers in Cardiovascular Medicine, 5. 888 https://doi.org/10.3389/fcvm.2018.00119

889 Mirth, C., \& Akam, M. (2002). Joint Development in the Drosophila Leg: Cell Movements and 890 Cell Populations. Developmental Biology, 246(2), 391-406. 891 https://doi.org/10.1006/dbio.2002.0593

892 Moczek, A. P., Sultan, S., Foster, S., Ledón-Rettig, C., Dworkin, I., Nijhout, H. F., Abouheif, E., 893 \& Pfennig, D. W. (2011). The role of developmental plasticity in evolutionary innovation. 894 Proceedings of the Royal Society B: Biological Sciences, 278(1719), 2705-2713. 895 https://doi.org/10.1098/rspb.2011.0971

896 Morgan, T. H. (1901). Regeneration. Macmillan and Co., Ltd.

897 Naviaux, R. K., Le, T. P., Bedelbaeva, K., Leferovich, J., Gourevitch, D., Sachadyn, P., Zhang, 898 X.-M., Clark, L., \& Heber-Katz, E. (2009). Retained Features of Embryonic Metabolism in the 899 Adult MRL Mouse. Molecular Genetics and Metabolism, 96(3), 133-144. 900 https://doi.org/10.1016/j.ymgme.2008.11.164

901 Nguyen, L.-T., Schmidt, H. A., von Haeseler, A., \& Minh, B. Q. (2015). IQ-TREE: A Fast and 902 Effective Stochastic Algorithm for Estimating Maximum-Likelihood Phylogenies. Molecular 903 Biology and Evolution, 32(1), 268-274. https://doi.org/10.1093/molbev/msu300 
904 Nicklin, P., Bergman, P., Zhang, B., Triantafellow, E., Wang, H., Nyfeler, B., Yang, H., Hild, 905 M., Kung, C., Wilson, C., Myer, V. E., MacKeigan, J. P., Porter, J. A., Wang, Y. K., Cantley, L. 906 C., Finan, P. M., \& Murphy, L. O. (2009). Bidirectional Transport of Amino Acids Regulates 907 mTOR and Autophagy. Cell, 136(3), 521-534. https://doi.org/10.1016/j.cell.2008.11.044

908 Ng, S., N., Zhu, H., de Soysa, T. Y., Shinoda, G., Seligson, M. T., Tsanov, K. M., Nguyen, L., 909 Asara, J. M., Cantley, L. C., \& Daley, G. Q. (2013). Lin28 enhances tissue repair by 910 reprogramming cellular metabolism. Cell, 778-792. 911 https://doi.org/10.1016/j.cell.2013.09.059

912 Rämet, M., Lanot, R., Zachary, D., \& Manfruelli, P. (2002). JNK Signaling Pathway Is Required 913 for Efficient Wound Healing in Drosophila. Developmental Biology, 241(1), 145-156. 914 https://doi.org/10.1006/dbio.2001.0502

915 Polezhaev, L. V. (1972). Loss and restoration of regenerative capacity in tissues and organs of 916 animals (Y. Halperin, Trans.). Harvard University Press.

917 Rouse, G. (1998). "The Annelida and their close relatives". In Anderson, D. T. (ed.). 918 Invertebrate Zoology. Oxford University Press.

919 Scorrano, S., Aglieri, G., Boero, F., Dawson, M., \& Piraino, S. (2017). Unmasking Aurelia 920 species in the Mediterranean Sea: An integrative morphometric and molecular approach. 921 Zoological Journal of the Linnean Society, 180, 243-267. https://doi.org/10.1111/zoj.12494

922 Simkin, J., Han, M., Yu, L., Yan, M., \& Muneoka, K. (2013). The Mouse Digit Tip: From 923 Wound Healing to Regeneration. In R. G. Gourdie \& T. A. Myers (Eds.), Wound Regeneration 924 and Repair (Vol. 1037, pp. 419-435). Humana Press. https://doi.org/10.1007/978-1-62703-505$925 \quad \underline{7} 24$

926 Singer, M. (1958). The regeneration of body parts. Scientific American, 199(4), 79-94.

927 Sinigaglia, C., Person, S., Eichelbrenner, J., Chevaler, S., Steger, J., Barreau, C., Houliston, E. \& 928 Leclere, L. (2020). Pattern regulation in a regenerating jellyfish. eLife 9, e54868. 929 https://doi.org/10.7554/eLife.54868

930 Sousonis, K., Baddour, J. A., \& Tsonis, P. A. (2014). Aging and regeneration in vertebrates. In 931 Current topics in developmental biology (Vol. 108, pp. 217-246). Academic Press.

932 Stipanuk, M. H. (2008). Leucine and Protein Synthesis: MTOR and Beyond. Nutrition Reviews, 933 65(3), 122-129. https://doi.org/10.1111/j.1753-4887.2007.tb00289.x

934 Takayama, K., Muto, A., \& Kikuchi, Y. (2018). Leucine/glutamine and v-ATPase/lysosomal 935 acidification via mTORC1 activation are required for position-dependent regeneration. Scientific 936 Reports, 8(1), 8278. https://doi.org/10.1038/s41598-018-26664-2

937 Togo, J., Hu, S., Li, M., Niu, C., \& Speakman, J. R. (2019). Impact of dietary sucrose on 938 adiposity and glucose homeostasis in C57BL/6J mice depends on mode of ingestion: Liquid or 939 solid. Molecular Metabolism, 27, 22-32. https://doi.org/10.1016/j.molmet.2019.05.010

940 Viechtbauer, W. (2010). Conducting Meta-Analyses in R with the metafor Package. Journal of 941 Statistical Software, 48.

942 Vieira, W. A., Wells, K. M., Raymond, M. J., De Souza, L., Garcia, E., \& McCusker, C. D. 943 (2019). FGF, BMP, and RA signaling are sufficient for the induction of complete limb 
944 regeneration from non-regenerating wounds on Ambystoma mexicanum limbs. Developmental 945 Biology, 451(2), 146-157. https://doi.org/10.1016/j.ydbio.2019.04.008

946 Vivien, C. J., Hudson, J. E., \& Porrello, E. R. (2016). Evolution, comparative biology and 947 ontogeny of vertebrate heart regeneration. Npj Regenerative Medicine, 1(1), 16012. 948 https://doi.org/10.1038/npjregenmed.2016.12

949 Schmid V. \& Alder, H. (1984). Isolated, mononucleated, striated muscle can undergo 950 pluripotent transdifferentiation and form a complex regenerate. Cell 38(3), 801-809. 951 https://doi.org/10.1016/0092-8674(84)90275-7

952 Wang, W., Hu, C.-K., Zeng, A., Alegre, D., Hu, D., Gotting, K., Ortega Granillo, A., Wang, Y., 953 Robb, S., Schnittker, R., Zhang, S., Alegre, D., Li, H., Ross, E., Zhang, N., Brunet, A., \& 954 Sánchez Alvarado, A. (2020). Changes in regeneration-responsive enhancers shape regenerative 955 capacities in vertebrates. Science, 369(6508), eaaz3090. https://doi.org/10.1126/science.aaz3090

956 West-Eberhard, M. J. (2003). Developmental plasticity and evolution. Oxford University Press.

957 Williams, M. C., Patel, J. H., Kakebeen, A. D. \& Wills, A. E. (2021). Nutrient availability 958 contributes to a graded refractory period for regeneration in Xenopus tropicalis. Developmental 959 Biology 473, 59-70. https://doi.org/10.1016/j.ydbio.2021.01.005

960 Wirtschafter, Z. T., \& Tsujimura, J. K. (1961). The sesamoid bones in long-evans strain rats. The 961 Anatomical Record, 141(3), 195-204. https://doi.org/10.1002/ar.1091410304

962 Wolfson, R. L., Chantranupong, L., Saxton, R. A., Shen, K., Scaria, S. M., Cantor, J. R., \& 963 Sabatini, D. M. (2016). Sestrin2 is a leucine sensor for the mTORC1 pathway. Science (New 964 York, N.Y.), 351(6268), 43-48. https://doi.org/10.1126/science.aab2674

965 Worley, M. I., Setiawan, L., \& Hariharan, I. K. (2012). Regeneration and Transdetermination in 966 Drosophila Imaginal Discs. Annual Review of Genetics, 46(1), $289-310$. 967 https://doi.org/10.1146/annurev-genet-110711-155637

968 Yu, L., Dawson, L. A., Yan, M., Zimmel, K., Lin, Y.-L., Dolan, C. P., Han, M., \& Muneoka, K. 969 (2019). BMP9 stimulates joint regeneration at digit amputation wounds in mice. Nature 970 Communications, 10(1). https://doi.org/10.1038/s41467-018-08278-4

971 Zhang, Y., Strehin, I., Bedelbaeva, K., Gourevitch, D., Clark, L., Leferovich, J., Messersmith, P. 972 B., \& Heber-Katz, E. (2015). Drug-induced regeneration in adult mice. Science Translational 973 Medicine, 7(290), 290ra92. https://doi.org/10.1126/scitranslmed.3010228 


\section{Supplementary Figures}

\begin{tabular}{|c|c|c|c|c|c|}
\hline Factor & $\begin{array}{l}\text { Highest } \\
\text { dose tested }\end{array}$ & Source & Factor & What was tested & Implementation \\
\hline \multicolumn{3}{|l|}{ Modulators of signaling pathways } & \multirow[t]{4}{*}{ Nutrient } & \multirow{4}{*}{$\begin{array}{l}1-50 \text { rotifers/animal } \\
0-5 \text { brine shrimps/animal } \\
\text { Combination of both }\end{array}$} & \multirow{4}{*}{$\begin{array}{l}\text { Food was administered } \\
\text { daily }\end{array}$} \\
\hline Erbstatin & $5 \mu \mathrm{M}$ & Sigma D2667 & & & \\
\hline hEGF recombinant & $20 \mathrm{ng} / \mathrm{mL}$ & Sigma E9644 & & & \\
\hline U0126 & $1 \mu \mathrm{M}$ & Millipore 6625 & & & \\
\hline Dorsomorphin & $1 \mu \mathrm{M}$ & Sigma P5499 & \multirow[t]{2}{*}{ Water current } & \multirow[t]{2}{*}{$0-60$ bubbles per minute } & \multirow{3}{*}{$\begin{array}{l}\text { Ambient air was pumped } \\
\text { to the cone }\end{array}$} \\
\hline $\mathrm{LiCl}$ & $250 \mathrm{mM}$ & Sigma L4408 & & & \\
\hline CHIR99021 & $12.5 \mu \mathrm{M}$ & Sigma SML1046 & \multirow{4}{*}{$\begin{array}{l}\text { Aquarium } \\
\text { geometry }\end{array}$} & \multirow{4}{*}{ Beaker, plate, tube, cone } & \\
\hline IWR-1 & $10 \mu \mathrm{M}$ & Sigma 10161 & & & \multirow{4}{*}{$\begin{array}{l}\text { Amputated ephyrae were } \\
\text { placed in different aquaria }\end{array}$} \\
\hline XAV939 & $2 \mu \mathrm{M}$ & Sigma X3004 & & & \\
\hline Purmorphamine & $2 \mu \mathrm{M}$ & Sigma SML0868 & & & \\
\hline hTGF- $\beta 1$ & $1.2 \mathrm{ng} / \mathrm{mL}$ & Peprotech 100-21 & Water volume & $100 \mathrm{~mL}-1 \mathrm{~L}$ & \\
\hline \multicolumn{3}{|c|}{ Modulators of metabolism, immune system, stress response } & \multirow[t]{2}{*}{ Animal density } & \multirow[t]{2}{*}{10 - 100 ephyrae / L } & \\
\hline Diosmetin & $10 \mu \mathrm{M}$ & Sigma D7321 & & & \\
\hline 17-DMAG & $1 \mu \mathrm{M}$ & TSZ Chemicals R1028 & \multirow{2}{*}{ Temperature } & \multirow{2}{*}{$18-25^{\circ} \mathrm{C}$} & \multirow{2}{*}{ Cooler or heater } \\
\hline Geranylgeranylacetone & $1 \mu \mathrm{M}$ & Sigma G5408 & & & \\
\hline KNK437 & $4 \mathrm{nM}$ & Sigma SML0964 & \multirow{3}{*}{ Heat shock } & $30 \mathrm{sec}$ at $42^{\circ} \mathrm{C}$ & \multirow{3}{*}{ Water bath } \\
\hline MKT-077 & $2.5 \mu \mathrm{M}$ & Sigma M5449 & & $30 \mathrm{~min}$ at $37^{\circ} \mathrm{C}$ & \\
\hline Bromopyruvic acid & $125 \mathrm{nM}$ & Sigma 16490 & & & \\
\hline 6-Phosphogluconic acid & $20 \mu \mathrm{M}$ & Sigma P7877 & \multirow[t]{3}{*}{ Dark } & \multirow{3}{*}{$\begin{array}{l}\text { Ephyrae were kept in in } \\
\text { the dark throughout the } \\
\text { experiment }\end{array}$} & \multirow{3}{*}{$\begin{array}{l}\text { Aquaria were wrapped } \\
\text { with aluminum foil }\end{array}$} \\
\hline Antamycin A & $650 \mathrm{nM}$ & Sigma A8674 & & & \\
\hline $3 \mathrm{PO}$ & $10 \mu \mathrm{M}$ & Millipore 525330 & & & \\
\hline ATP & $5 \mu \mathrm{M}$ & Sigma A3377 & \multirow{4}{*}{ Salinity } & \multirow{4}{*}{$18-55 \mathrm{ppt}$} & \multirow{6}{*}{$\begin{array}{l}\text { Ephyrae were placed in } \\
\text { artificial seawater with } \\
\text { varying salinity } \\
\text { Incubator, } 60-120 \mathrm{rpm} \\
\text { Rottiserrie, 5-8 rpm } \\
\text { Air tubing, } 0-60 \text { bubbles/min }\end{array}$} \\
\hline 3BDO & $3 \mu \mathrm{M}$ & Sigma SML1687 & & & \\
\hline D-Fructose 1.6-bisphosphate & $20 \mu \mathrm{M}$ & Sigma F6803 & & & \\
\hline DMOG & $50 \mu \mathrm{M}$ & Millipore 400091 & & & \\
\hline Rapamycin & $1 \mu \mathrm{M}$ & Sigma R8781 & \multirow{2}{*}{$\begin{array}{l}\text { Means of } \\
\text { current } \\
\text { generation }\end{array}$} & \multirow{10}{*}{$\begin{array}{l}\text { Shaking } \\
\text { Rotating } \\
\text { Bubbling }\end{array}$} & \\
\hline $\begin{array}{l}\text { L-Leucine methyl esther } \\
\text { hydrochloride (cell permeable } \\
\text { form) }\end{array}$ & $100 \mu \mathrm{M}$ & Sigma L1002 & & & \\
\hline Resveratrol & $5 \mu \mathrm{M}$ & Sigma R5010 & & & \\
\hline Sapanisertib & $2 \mathrm{nM}$ & Selleck Chemicals S2811 & & & \\
\hline MHY1485 & $2 \mu \mathrm{M}$ & Sigma SML0810 & & & \\
\hline Insulin, human & $3 \mu \mathrm{M}$ & Sigma 10908 & & & \\
\hline AICAR & $25 \mu \mathrm{M}$ & Santa Cruz sc-200659A & & & \\
\hline A769662 & $5 \mu \mathrm{M}$ & Santa Cruz sc-203790 & & & \\
\hline D-Eryhtrose 4-phosphate & $20 \mu \mathrm{M}$ & Sigma E0377 & & & \\
\hline $\mathrm{CoCl}$ & $450 \mathrm{nM}$ & Sigma 60818 & & & \\
\hline Miscellaneous & & & & & \\
\hline BSA & $500 \mathrm{nM}$ & Sigma A7906 & & & \\
\hline Ethanol & $20 \mu \mathrm{L} / \mathrm{L}$ & VWR 89125-170 & & & \\
\hline $\mathrm{CsCl}$ & $5 \mu \mathrm{L} / \mathrm{L}$ & Sigma C4036 & & & \\
\hline
\end{tabular}

Figure 2-figure supplement 1. Various molecular and physical modulations were screened to recapitulate arm regeneration.

978 The experimental design was as described in Methods for the main experiments. Essentially, the

979 screen methodology was as follow:

980 A. The choice of the factors screened was dictated by a combination of considerations:

981 - Evidence in literature for involvement in regeneration in other systems, e.g., various 982 developmental pathways

983 - Natural factors that have been shown, or are intuitively would be, relevant to Aurelia's life

984 history, e.g., nutrient level, oxygen level.

985 - Limitations such as availability for drugs or feasible implementation for physical factors.

986 The specific rationale for each factor tested is detailed in the source file 'Aurelia screen.xls'. 
987 B. Experimental design and regeneration assessment. Experiments were performed at $20^{\circ} \mathrm{C}$, 988 except for those testing the effects of different temperatures. Two-to-three-day-old ephyrae were 989 anesthetized in $400 \mu \mathrm{M}$ menthol and amputated using a razor blade. In all experiments, the 990 amputation removed three arms, as illustrated in Figure 1c. Except for experiments testing 991 aquaria setups, amputated ephyrae were let to recover in $1 \mathrm{~L}$ sand settling cones (Nalgene 992 Imhoff, Figure 3-figure supplement 1). In each experiment, control and treated groups were set 993 up side by side. Control and treated ephyrae were fed daily. Each experiment was repeated 994 across 2-5 strobilation batches (biological replicates). Regeneration was assessed at 1-2 weeks 995 after amputation.

996 C. Modulators or changes in physical parameters were implemented upon amputation. Small 997 molecules were refreshed weekly. The drug targets, the concentrations tested, and the number of 998 ephyra tested are detailed in the source file 'Aurelia screen.xls'. Some factors were dissolved in 999 DMSO or ethanol; for these molecules, the control group was administered with an equal volume 1000 of the solvent.

1001 D. To determine the range of parameters tested, since few, if at all, of the molecular 1002 modulators had been tested in Aurelia, the maximum concentrations were tested to maximize the 1003 chance of seeing an effect. Maximum concentration was determined by solubility in saltwater or 1004 onset of adverse effects (e.g., degrowth, paralysis, death) upon overnight incubation. Where 1005 available, previously reported concentrations in cell culture or animal systems were included in 1006 the testing. A negative result means no obvious effects were observed at the maximum 1007 concentration that warrant further investigation. For factors that gave interesting effects (e.g., 1008 insulin), a range of lower concentrations were subsequently tested for optimization. 


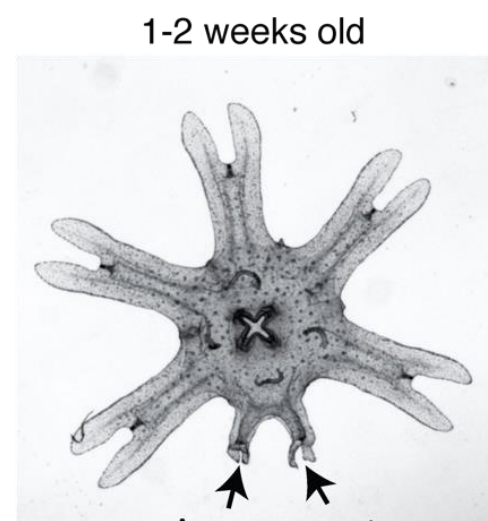

Arm regenerates are obvious

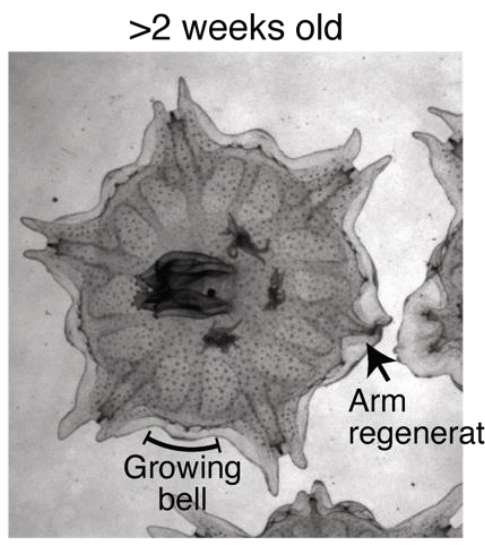

The arm regenerate is beginning to be masked by the growing bell.

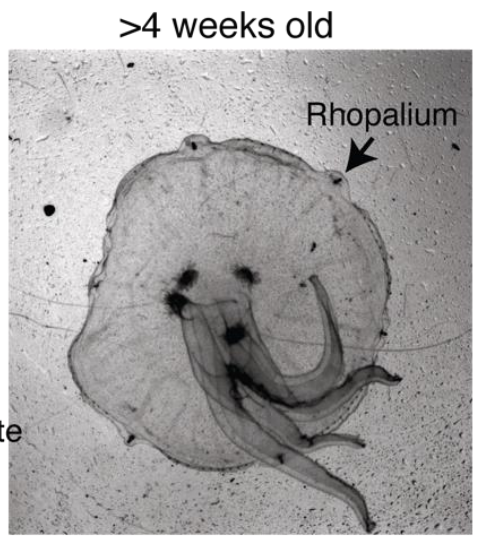

Discrete arms are no longer visible as tissues have grown to fill the space between the arms. Rhopalia count can be used as a proxy for scoring arm regenerates, but not all arm regenerates develop rhopalia.

1010 Figure 2-figure supplement 2. Bell growth limited the time window for assessing arm 1011 regeneration.

1012 Ephyrae in the lab mature into full-belled medusae within $\sim 4$ weeks. The transition to medusa 1013 commences at 1-2 weeks after strobilation, with the onset of bell growth. Over 2-3 weeks, body 1014 tissues gradually grow and fill between the discrete arms to form a continuous bell characteristic 1015 of a medusa. Arm regeneration can be unambiguously scored in ephyrae before the bell has 1016 significantly grown. Bell growth also limited testable doses in some factors, e.g., testing higher 1017 food amounts than reported here led to accelerated bell growth at a rate that did not allow enough 1018 time window to quantify regeneration. 
a Original, wild
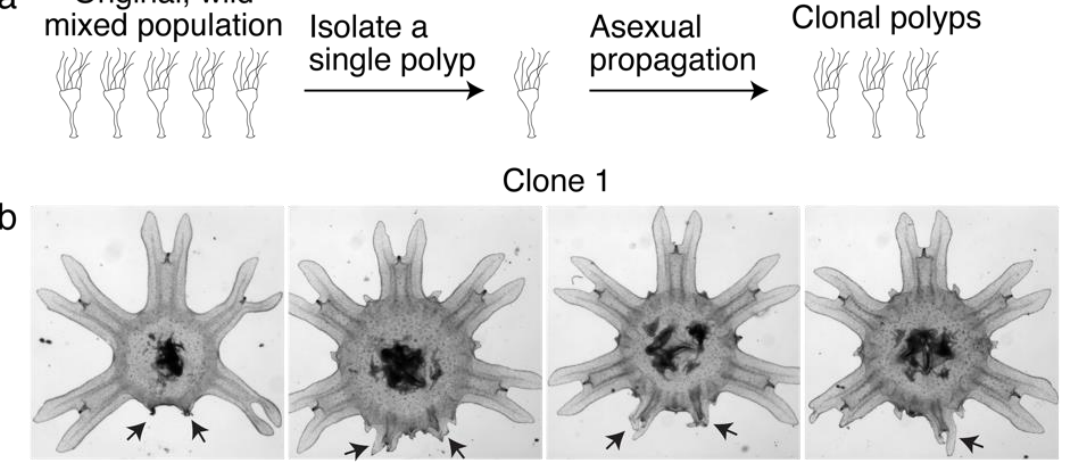

Clone 3

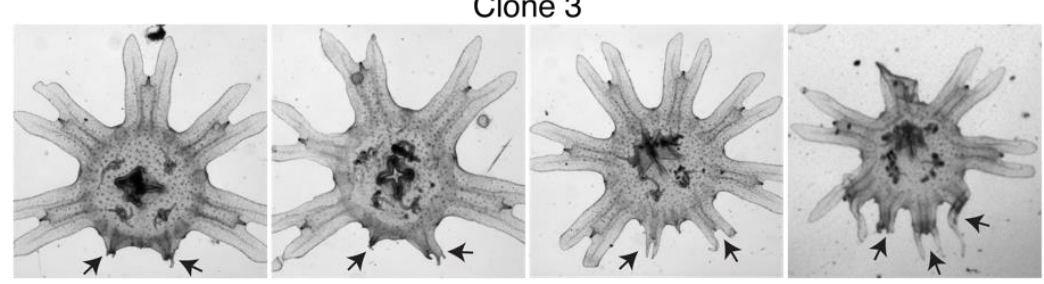

C Regeneration frequency (\%)

Figure 2-figure supplement 3. Variable extent of regeneration was observed in clonal lines.

(a) To develop genetically clonal lines, single polyps were isolated and settled onto tissue culture dishes. Within 1-3 months, with daily feeding of enriched brine shrimps, each dish was repopulated with polyps asexually budding from the single parental polyp.

1026 (b) Regeneration induction with high food performed in two clonal lines. Arrows indicate arm regenerates.

1028

1029

(c) Regeneration frequency in the clonal and original mixed populations measured in the same

1030 experiment.

1031 


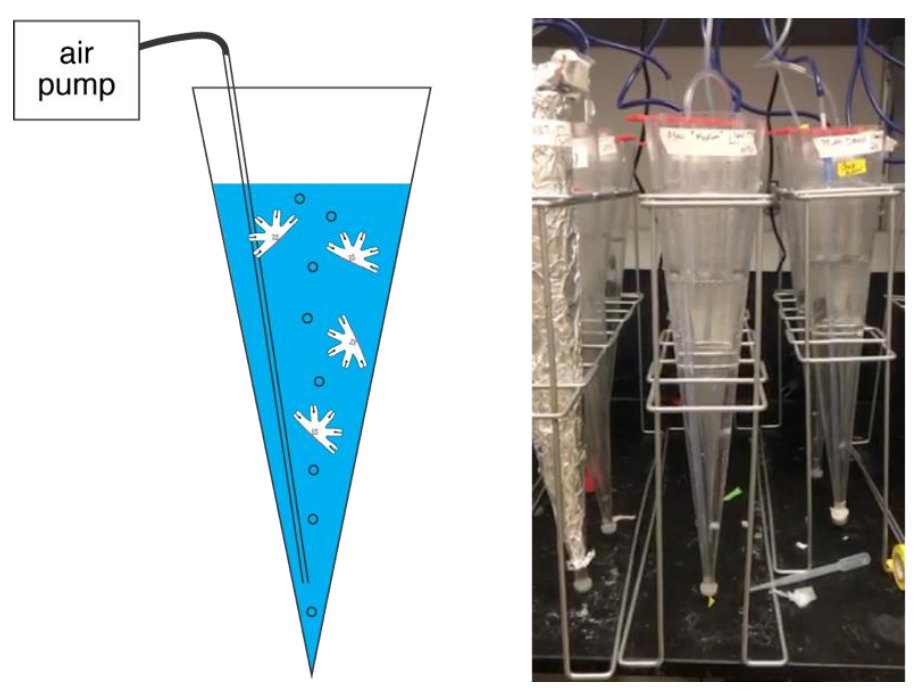

1033 Figure 3-figure supplement 1. Water current is a permissive requirement for arm 1034 regeneration induction.

1035 Various physical environments for the ephyrae recovering from injury were tested, e.g., shallow 1036 vs deep water, seawater with varying salinity, cold vs warm temperature, light versus dark, 1037 stagnant water vs current, generating water current through various means, including shaking or 1038 rotating to generate turbulent mixing and as shown here air bubbling a conical tube to generate 1039 vertical current (shown here). While symmetrization occurred robustly in all conditions, 1040 consistent induction of regeneration only occurred in the presence of columnar water current. 1041 The experiments presented in this study were performed in the "bubbler cone" setup, where a 1L 1042 sand settling cone was repurposed into an aquarium and connected to an air pump (Tetra 1043 Whisper 100) to generate a gentle current of $\sim 1$ bubble/second (Video 2). Each cone housed 30 1044 ephyrae in $500 \mathrm{~mL}$ ASW or treated ASW, refreshed weekly, to avoid crowding and fouling. In 1045 the bubbler cone, ephyrae continually move along water current, either the upward bubble1046 generated current or the downward gravity-generated current. The conical geometry helps 1047 minimize stagnant spots, where the ephyrae could get stuck. 


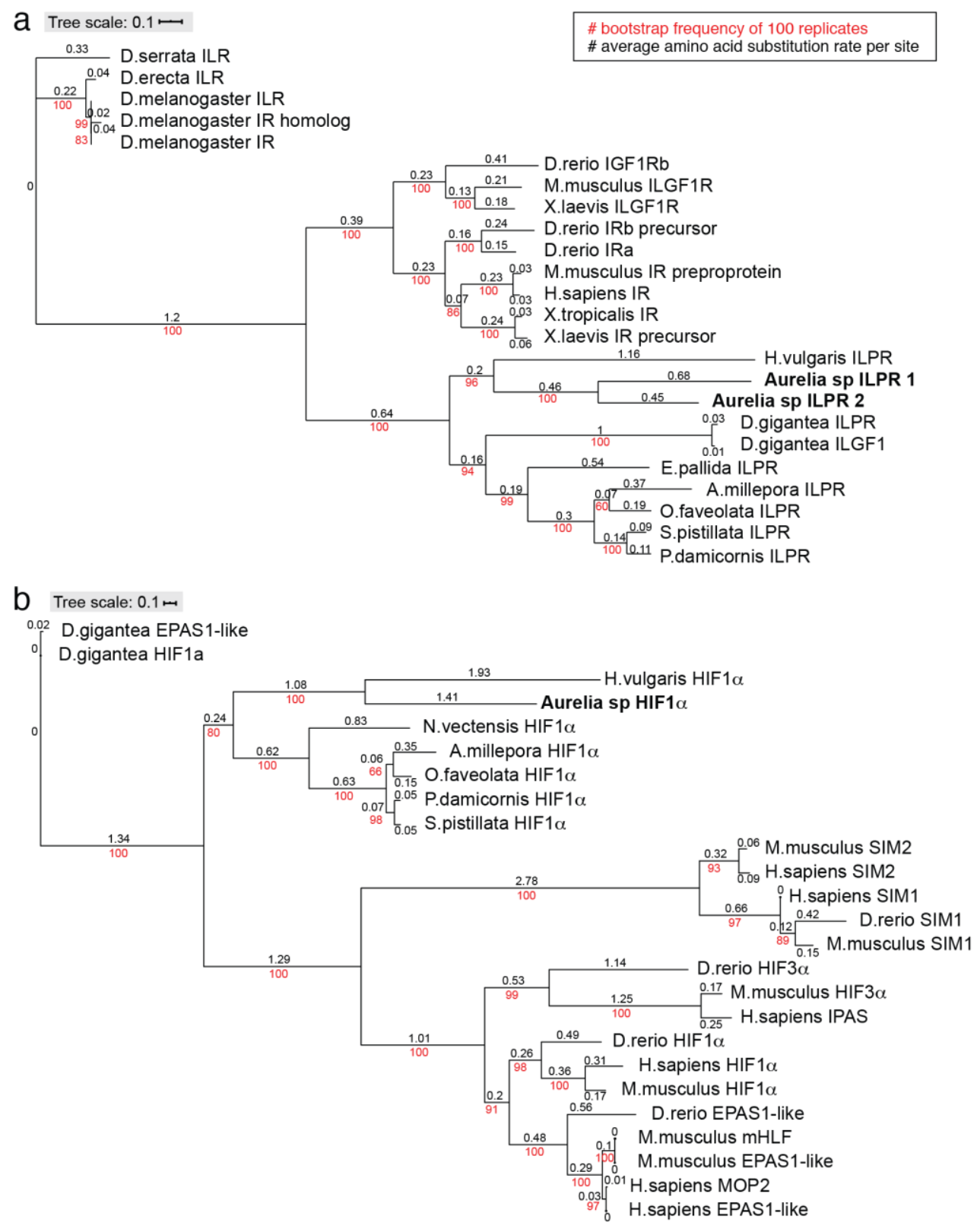

1050 Figure 3-figure supplement 2. Conservation of insulin receptor and HIFa in Aurelia.

1051 Phylogenies of insulin receptor (a) and HIFa (b) genes were constructed using the maximum 1052 likelihood inference computed with the IQ-TREE stochastic algorithm (Nguyen et al., 2015), and 1053 visualized using ITOL (https://itol.embl.de/upload.cgi). These simple trees are not meant to be 1054 comprehensive, but a verification of the genes annotated as insulin-like protein receptor (ILPR) 1055 and HIF $\alpha$ in the Aurelia gene models by testing conservation with their known counterparts in 1056 other organisms. IQ-TREE parameters: Insulin receptor consensus tree is constructed from 1000 1057 bootstrap trees; log-likelihood of consensus tree is -45374.0; the Robinson-Foulds distance 1058 between ML and consensus tree is 0 . HIF $\alpha$ consensus tree is constructed from 1000 bootstrap 1059 trees; log-likelihood of consensus tree is -24414.4; the Robinson-Foulds distance between ML 1060 and consensus tree is 0. 


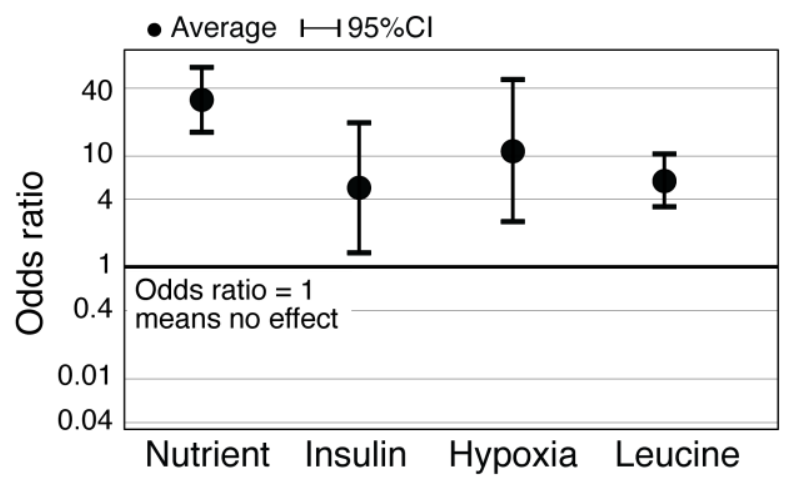

\begin{tabular}{|lllll|}
\hline Treatment & Odds ratio & $\mathbf{9 5 \%} \mathbf{C l}$ & p-value \\
Nutrient & 30.9 & $15.8,60.6$ & $<0.0001$ & *** \\
Insulin & 5.0 & $1.3,19.4$ & 0.02 & * \\
Hypoxia & 10.8 & $2.5,46,6$ & 0.001 & $* \star$ \\
Leucine & 5.8 & $3.4,10.1$ & $<0.0001$ & $* \star *$ \\
\hline
\end{tabular}

1063 Figure 3-figure supplement 3. Statistical significance of regeneration induction in Aurelia 1064 assessed using Odds Ratio.

In addition to RR analysis presented in Figure $3 \mathrm{~g}$, another common measure of effect size is the Odds Ratio (OR) (Borenstein et al., 2009). OR compares the odds of outcome in the presence vs. absence of treatment (Methods). Analysis of OR across experiments was performed using the metafor package (Viechtbauer, 2010) in $\mathrm{R}$ with statistical coefficients based on normal distribution (Methods). A treatment is reproducible if the $95 \%$ confidence intervals (95\% CI) exclude 1. The p-value evaluates the null hypothesis that the estimate OR is 1. 

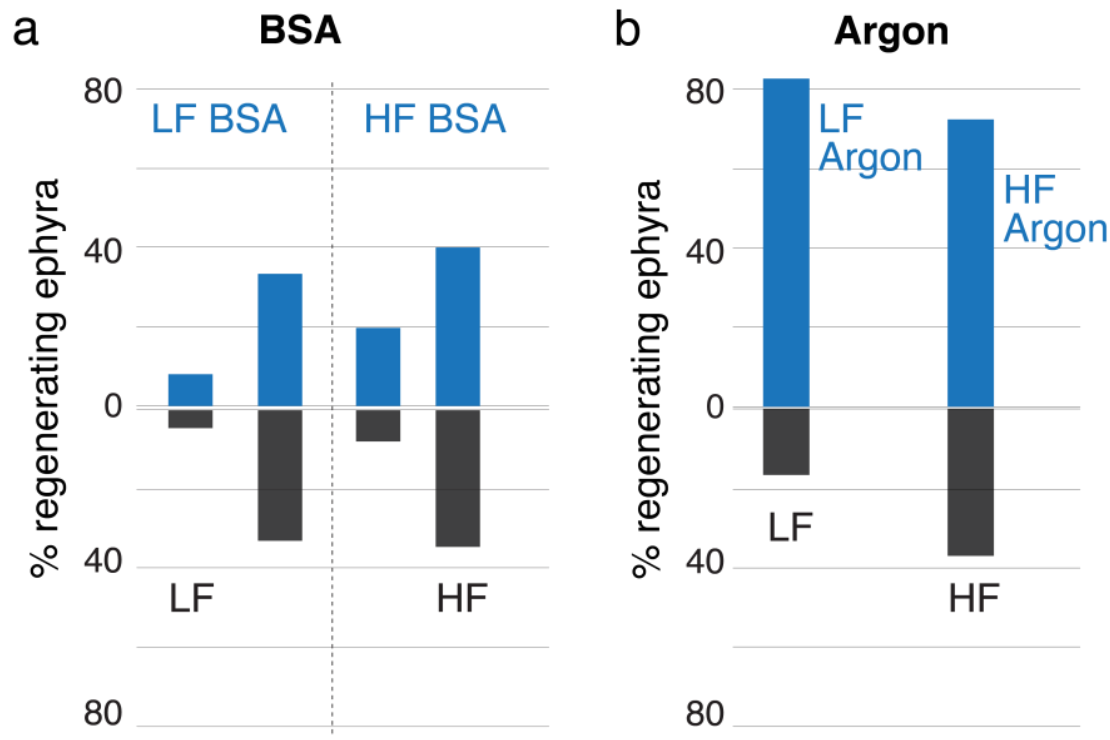

$\begin{array}{rcccc}\text { Exp } & 1 & 2 & 3 & 4 \\ \% & 4.5 & 32.9 & 7.9 & 34.5 \\ & 8.0 & 33.3 & 19.7 & 40.0 \\ \mathbf{N} & 89 & 85 & 89 & 58 \\ & 25 & 45 & 66 & 30\end{array}$

$\begin{array}{rcc}\text { Exp } & 1 & 2 \\ \% & 16.7 & 37.1 \\ & 82.8 & 72.5 \\ \mathbf{N} & 90 & 89 \\ & 87 & 69\end{array}$

1074 Figure 3-figure supplement 4. Treatments using bovine serum albumin and argon.

1075 (a) Treatment with $500 \mathrm{nM}$ bovine serum albumin (BSA) did not produce significant effect in 1076 regeneration frequency $(95 \% \mathrm{CI}[0.9,1.9$-fold change], p-value=0.20).

1077 (b) Reducing oxygen using argon flow increased regeneration frequency (95\% CI [1.99, 3.3-fold 1078 change], p-value $<10^{-4}$ ).

1079 Effect size was computed using the metric Risk Ratio (see Methods). LF is low food, HF is high 1080 food, Exp is Experiment ID, \% is percentage of regenerating ephyra in control (black) and 1081 treated (blue), $\mathbf{N}$ is the number of ephyrae in the control (black) and treated (blue) group. 


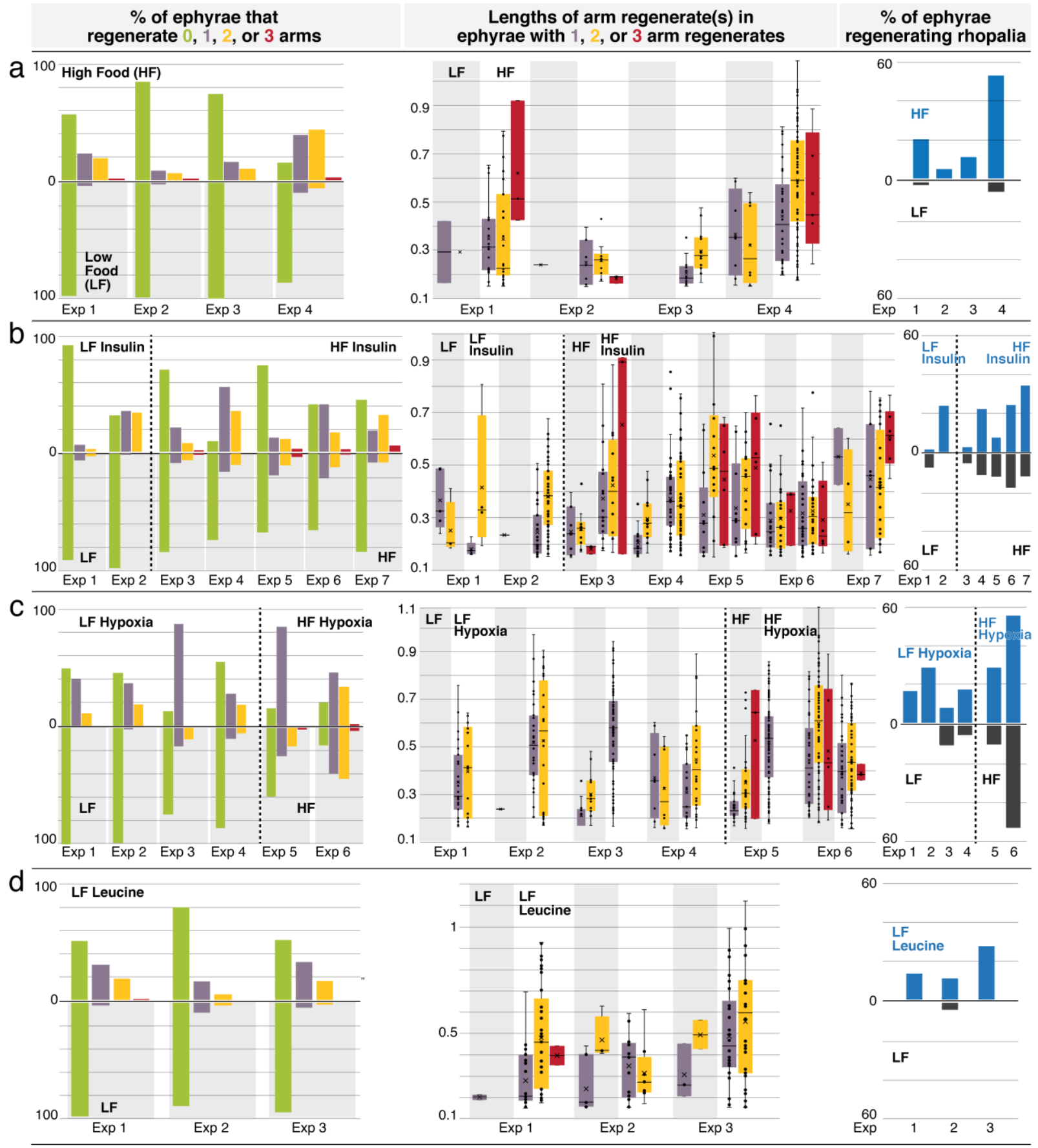

Figure 3-figure supplement 5. Regeneration phenotypes. Left: The percentage of ephyrae that regenerate 0 (green), 1 (purple), 2 (yellow), or 3 arms (red). Middle: The length(s) of arm regenerate(s) in ephyrae that regenerate 1 arm (purple), 2 arms (yellow), and 3 arms (red) - normalized to the average length of uncut arms in the same ephyra. For ephyrae with multiple arm regenerates, lengths of all arms were measured and plotted individually. Boxplot: median (line), average (cross), 1st and 3rd quartiles (the box), $5^{\text {th }}$ and $95^{\text {th }}$

1091 percentile (whiskers), and individual data points (black circles).

1092 Right: Percentage of ephyrae that reform rhopalia in control (grey) and treated (blue) groups. 
$\%$ ephyrae regenerating

$\%$ ephyrae regenerating $>1$ arm

Length of arm regenerates

$\%$ ephyrae regenerating rhopalia

$\%$ ephyrae regenerating

$\%$ ephyrae regenerating $>1$ arm

Length of arm regenerates

$\%$ ephyrae regenerating rhopalia
High food

\begin{tabular}{|lll|}
\hline $\begin{array}{l}\text { Effect } \\
\text { size }\end{array}$ & $95 \% \mathbf{C l}$ & p-value \\
7.5 & $4.7,12.1$ & $<0.0001^{* * *}$ \\
11.4 & $4.9,26.4$ & $<0.0001^{* * *}$ \\
1.6 & $1.2,2.0$ & $0.0003^{* * *}$ \\
11.8 & $5.3,26.5$ & $<0.0001^{* * *}$ \\
\hline
\end{tabular}

\begin{tabular}{|llll|}
\multicolumn{5}{c|}{ Insulin } \\
\hline $\begin{array}{l}\text { Effect } \\
\text { size }\end{array}$ & $\mathbf{9 5 \%} \mathbf{C l}$ & p-value \\
2.4 & $1.1,5.0$ & 0.023 & * \\
1.9 & $1.3,2.8$ & 0.0005 & $* \star \star$ \\
1.2 & $0.98,1.5$ & 0.080 & n.s \\
1.3 & $0.7,2.7$ & 0.427 & n.s \\
\hline
\end{tabular}

L-leucine

$\begin{array}{lll}\begin{array}{l}\text { Effect } \\ \text { size }\end{array} & 95 \% \text { Cl } & \text { p-value } \\ 4.1 & 2.5,6.6 & <0.0001 \\ 6.0 & 1.9,19.1 & 0.003 \quad \text { ** } \\ 1.7 & 1.4,1.9 & <0.0001^{* * *} \\ 6.1 & 2.1,17.7 & 0.00099^{* * *}\end{array}$

Hypoxia

\begin{tabular}{|llll|}
\hline $\begin{array}{l}\text { Effect } \\
\text { size }\end{array}$ & $\mathbf{9 5 \%} \mathbf{C l}$ & \multicolumn{2}{l|}{ p-value } \\
4.1 & $1.4,12.0$ & 0.0099 & ** \\
1.2 & $0.2,9.0$ & 0.833 & n.s \\
1.3 & $0.8,2.1$ & 0.239 & n.s \\
2.6 & $1.01,6.7$ & 0.047 & * \\
\hline
\end{tabular}

1094 Figure 3-figure supplement 6. Statistical analysis of the regeneration phenotypes in high amount of nutrients, insulin, hypoxia, and L-leucine. For frequency measurements, the effect size of a treatment compares the probability of an outcome in treated vs. control group (i.e., Risk Ratio, Methods). For length measurement, the effect size of a treatment compares the proportionate change that results from the treatment (i.e., Response Ratio, Methods). Analysis of effect size across experiments was performed using the metafor package15 in $\mathrm{R}$ with statistical coefficients based on normal distribution (Methods). A treatment is reproducible if the $95 \%$ confidence intervals $(95 \% \mathrm{CI})$ exclude 1 . The p-value evaluates the null hypothesis that the estimate effect size is 1 (i.e., no effect).

1103 Source files: Aurelia regeneration data.xls; R-codes.rtf 

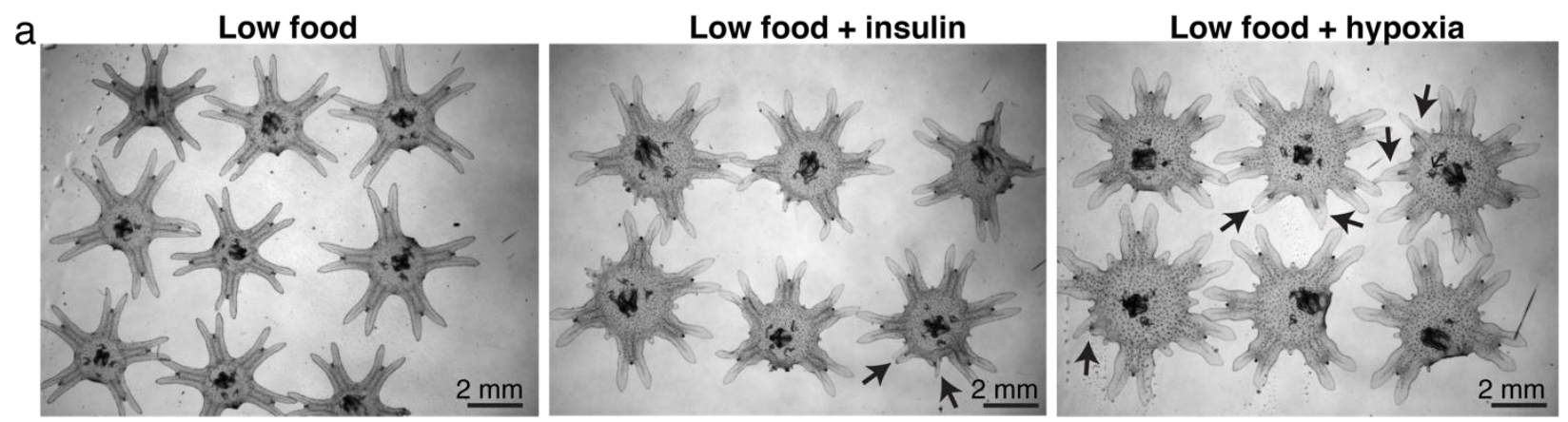

b

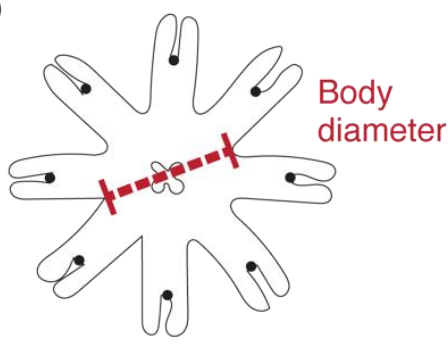

1105

1106

1107

1108

1109

1110

1111

1112

1113

1114

\begin{tabular}{|lcll|}
\hline Treatment & $\begin{array}{c}\text { Ave. body diameter } \\
\text { treatment/control }\end{array}$ & $95 \% \mathrm{Cl}$ & p-value \\
High food & 1.7 & $1.6,1.8$ & $<0.0001^{\text {** }}$ \\
Insulin & 1.4 & $1.1,1.8$ & $0.011^{*}$ \\
Hypoxia & 1.5 & $1.3,1.9$ & $<0.0001^{\text {** }}$ \\
Leucine & 1.1 & $1.04,1.12$ & $<0.0001^{\text {** }}$ \\
\hline
\end{tabular}

Figure 3-figure supplement 7. Ephyrae in high food, insulin, or hypoxia, and L-leucine tend to be bigger in size.

(a) Representative images of ephyrae growing in low food, $500 \mathrm{nM}$ insulin, and hypoxia. Black arrows indicate regenerating arms.

(b) Effect size analysis of the body size increase was performed using the metafor package (Viechtbauer et al., 2010) in R (Methods). A treatment effect is reproducible if the 95\% CI exclude 1 . The p-value evaluates the hypothesis that there is no effect.

Source files: Aurelia regeneration data.xls; R-codes.rtf 
a

\begin{tabular}{lllll}
\hline Strain & Condition & $\mathrm{N}$ & No clot & Regrown tibia \\
\hline Oregon R & Control & 860 & $0 \%$ & $0 \%$ \\
Oregon R & Leucine \& Insulin & 387 & 12.1 & $1.0 \%$ \\
Canton S & Control & 174 & $0 \%$ & $0 \%$ \\
Canton S & Leucine \& Insulin & 284 & $29.9 \%$ & $1.1 \%$ \\
\hline
\end{tabular}

b

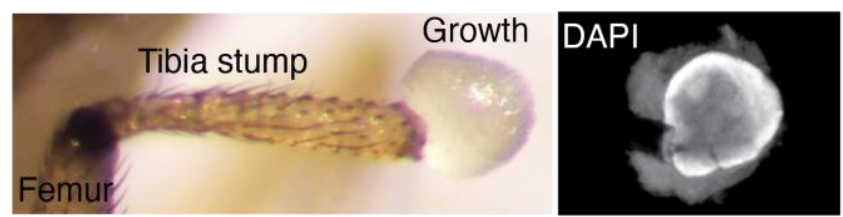

1116 Figure 4-figure supplement 1. Regenerative response induced in Drosophila was observed 1117 across wild-type strains.

1118 (a) Summary of the bulk experiment data in Oregon R and Canton S wild-type strains.

1119 (b) Few flies showed non-patterned outgrowth from the amputation site (3 out of 387 in Oregon 1120 R, 9 out of 284 in Canton S). 

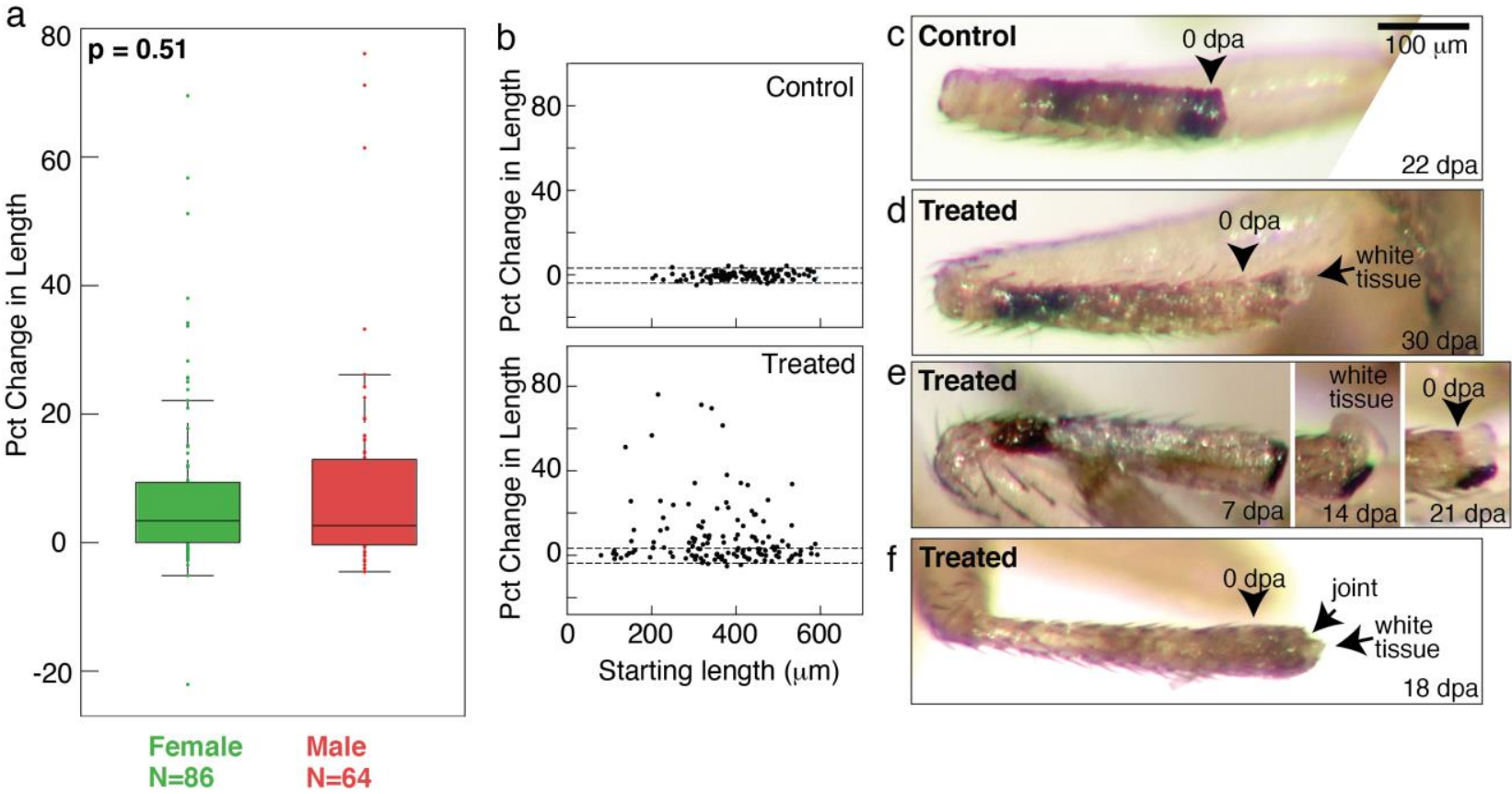

1123 Figure 5-figure supplement 1. Tracking regenerative response in single flies.

1124 (a) No sex-based differences were observed in the regenerative response. Distributions of 1125 changes in stump lengths in male and female flies are not statistically different ( $\mathrm{p}$-value $=0.48$, 1126 non-parametric Kruskal-Wallis test).

1127 (b) Growth was observed from proximal (short tibia stump) or distal (long tibia stump) 1128 amputation across tibia.

1129 (c) A control tibia stump.

1130 (d-f) Treated tibia stumps showing white tissues growing from the end. 

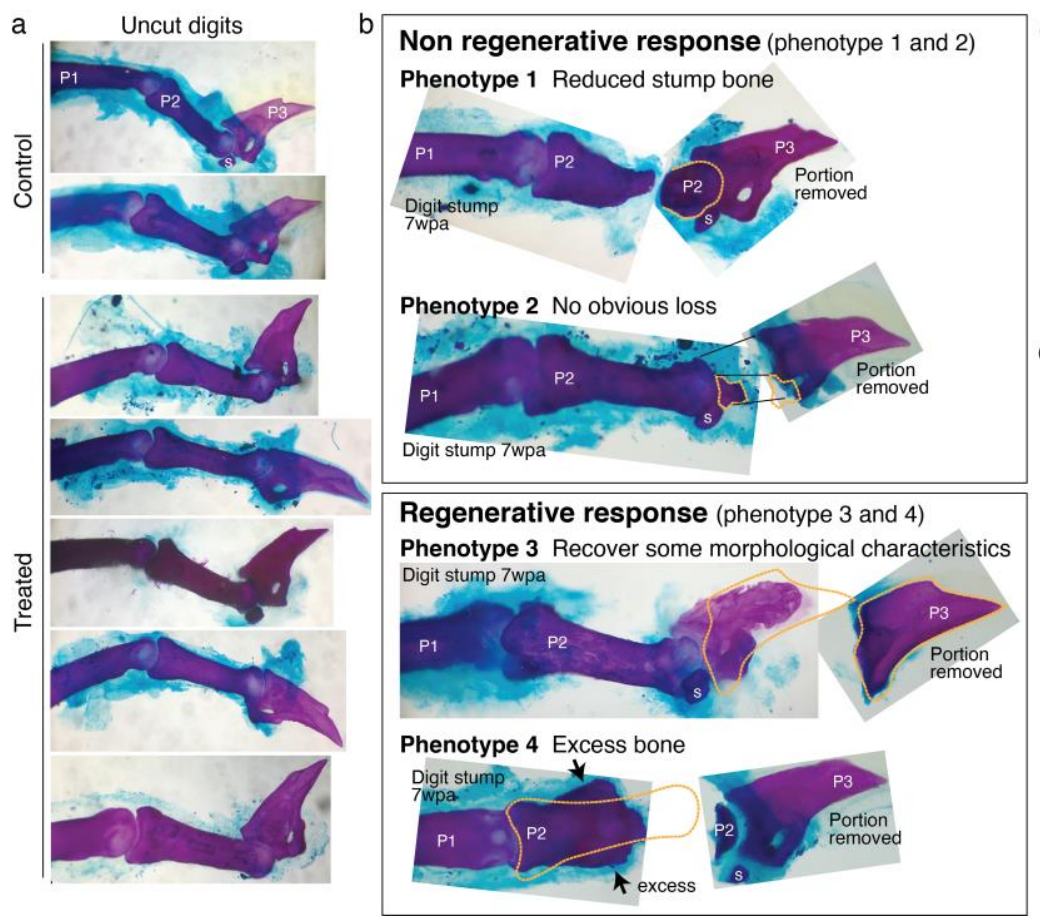

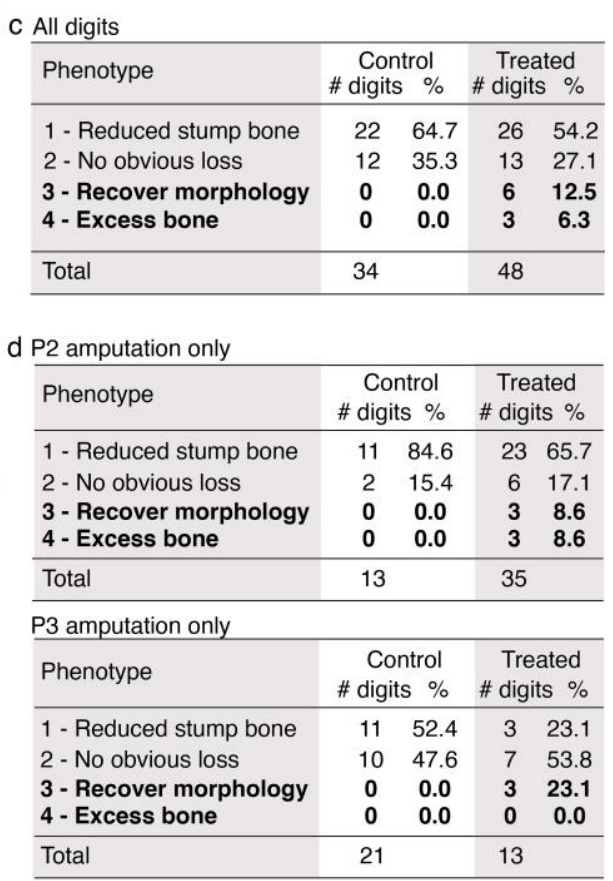

\section{Figure 6-figure supplement 1. Mouse digit phenotypes.}

1134 Whole-mount skeletal staining was performed with Alizarin red. wpa: week post amputation, P1: 1135 phalange 1, P2: phalange 2, P3: phalange 3, s: sesamoid bone

1136 (a) Skeletal staining of unamputated digits (digit 3) from control and treated groups show no obvious differences in uncut digits due to the treatment.

1138 (b) Skeletal staining of digits stumps at $7 \mathrm{wpa}$ and the original portion removed from the digits. 1139 Some digit stumps show no change or appear to have undergone histolysis (Chamberlain et al. 1140 2017) resulting in reduced bone mass (Phenotype 1 and 2). Some digit stumps show regenerative 1141 response, either recovery of some morphological characteristics (Phenotype 3, detailed more in 1142 Figure 6-figure supplement 2) or excess, ectopic bone mass (Phenotype 4). We erred on the 1143 conservative side in scoring phenotype 3 and 4; when in doubt, digits were classified into 1144 phenotype 1 or 2.

1145 (c-e) Phenotype counts in all digits (c), in digits amputated across P2 (d), and in digits amputated 1146 across P3 or joint (e).

1147 Source file: Mouse data.xls 


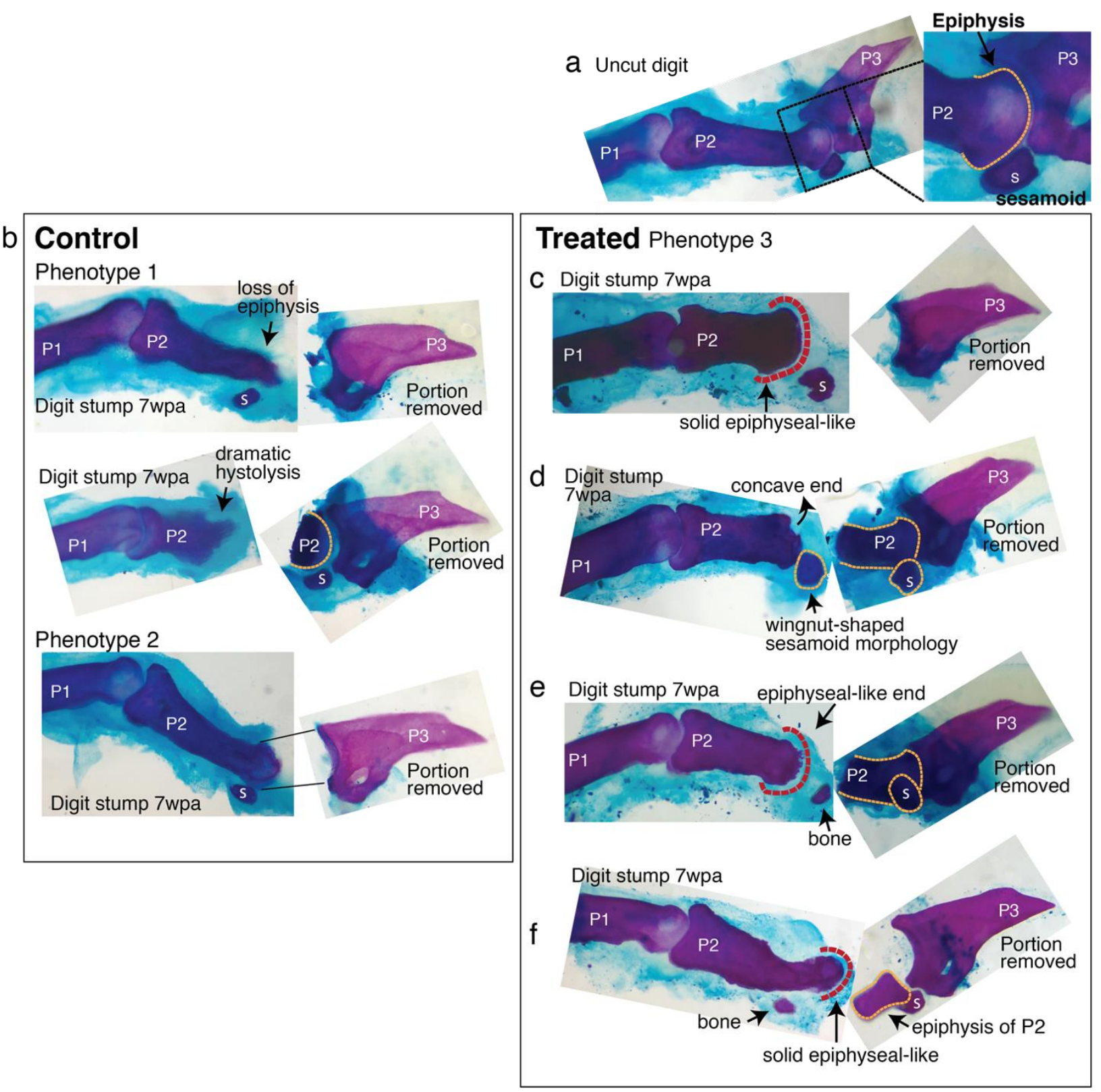

Control

Phenotype

Phenotype 2

Figure 6-figure supplement 2. Regenerative response observed in mouse digit.

1151 Six digit stumps (of total 48 examined) show regenerative response. The most dramatic two are 1152 presented in Figure 4. The remaining four are presented here.

1153 wpa: week post amputation, P1: phalange 1, P2: phalange 2, P3: phalange 3, s: sesamoid bone

1154 (a) An uncut digit, shown for a comparison. Magnified is the P2/P3 joint area to highlight key 1155 morphological markers: the knobby epiphyseal cap of P2 and the sesamoid bone embedded in 1156 the tendon on the flexor side of P2.

1157 (b) Digit stumps from control mice show either bone stump histolysis (top and middle, 1158 phenotype 1) and no visible changes in bone stump (bottom, phenotype 2).

1159 (c-f) Digit stumps from treated mice that show regenerative response. 
1160 (c) In this digit, the amputation removed all P3 by a cut through the joint. At 7 wpa, the P2 stump 1161 is reduced, but recovered the epiphyseal-like end (red dashed line) - marked by solid curved 1162 shape, as opposed to irregularly shaped histolyzing bone.

1163 (d) In this digit, the amputation removed a significant portion of P2 and the sesamoid bone. The 1164 P2 stump does not regain an epiphyseal end (the end is concave and irregular). However, the 1165 sesamoid bone is reformed, as identified by its location on the flexor side of P2 and wingnut 1166 shape (Wirtschafter and Tsujimura, 1961) under the microscope. The recovery of sesamoid bone 1167 is non-trivial, as digit sesamoids form in juxtaposition to the condensing phalange, detaching 1168 from the phalange by formation of a cartilaginous joint (Eyal et al. 2019).

1169 (e) In this digit, the amputation removed a significant portion of P2 and the sesamoid bone. At 7 1170 wpa, the P2 stump appears to be reforming an epiphyseal, rounded end (red dashed line). There 1171 is a small bone distal to P2, whose curvature articulates with the P2 end, but there are not enough 1172 morphological characters to identify the bone.

1173 (f) In this digit, the amputation removed the epiphyseal cap of P2 and the sesamoid bone. The P2 1174 stump appears to have lost some mass, but reforms an epiphyseal-like end (red dashed line). 1175 There is an additional small bone located where the sesamoid bone should be, but lacks sufficient 1176 morphological characters to identify. 